\title{
The additivity of the $\rho$-invariant and periodicity in topological surgery
}

\author{
DIARMUID CROWLEY \\ TIBOR MACKO
}

For a closed topological manifold $M$ with $\operatorname{dim}(M) \geq 5$ the topological structure set $\mathcal{S}(M)$ admits an abelian group structure which may be identified with the algebraic structure group of $M$ as defined by Ranicki. If $\operatorname{dim}(M)=2 d-1, M$ is oriented and $M$ is equipped with a map to the classifying space of a finite group $G$, then the reduced $\rho$-invariant defines a function,

$$
\tilde{\rho}: \mathcal{S}(M) \rightarrow \mathbb{Q} R_{\widehat{G}}^{(-1)^{d}},
$$

to a certain subquotient of the complex representation ring of $G$. We show that the function $\tilde{\rho}$ is a homomorphism when $2 d-1 \geq 5$.

Along the way we give a detailed proof that a geometrically defined map due to Cappell and Weinberger realises the 8-fold Siebenmann periodicity map in topological surgery.

$57 \mathrm{R} 65,57 \mathrm{~S} 25$

\section{Introduction}

Let $M$ be a closed oriented $(2 d-1)$-dimensional topological manifold and let $\lambda(M): M \rightarrow B G$ be a map to the classifying space of a finite group $G$. The $\rho-$ invariant of $(M, \lambda(M))$,

$$
\rho(M, \lambda(M)) \in \mathbb{Q} R_{\widehat{G}}^{(-1)^{d}},
$$

lies in a certain subquotient of the rationalised complex representation ring of $G$ (see Section 2.1 for details). It is a powerful invariant of odd-dimensional manifolds with torsion elements in their fundamental group. To mention just two examples: it was used by Atiyah and Bott to show that two smooth lens spaces which are $h$-cobordant are diffeomorphic [1]. It also plays a key role in Wall's classification results for fake lens spaces in the piecewise linear and topological categories [32, Chapter 14].

Assume now that $2 d-1 \geq 5$ and consider $\mathcal{S}(M)$, the topological structure set of $M$. The elements of $\mathcal{S}(M)$ are homotopy equivalences $h: N \rightarrow M$ of closed manifolds 
modulo the $h$-cobordism relation in the source. ${ }^{1}$ We define the reduced $\rho$-invariant by

$$
\tilde{\rho}: \mathcal{S}(M) \longrightarrow \mathbb{Q} R_{\widehat{G}}^{(-1)^{d}}, \quad[h: N \rightarrow M] \longmapsto \rho(N, \lambda \circ h)-\rho(M, \lambda) .
$$

A feature of topological surgery is that $\mathcal{S}(M)$ admits the structure of an abelian group which is natural in some sense; see Siebenmann [31] and Ranicki [23]. Since this group structure on $\mathcal{S}(M)$ is mysterious from the geometric point of view it is not clear whether $\tilde{\rho}$ is a homomorphism of abelian groups.

It is clear, however, that $\tilde{\rho}$ is additive with respect to the action of the $L$-group on $\mathcal{S}(M)$. Let $\pi=\pi_{1}(M)$ and recall that the surgery group $L_{2 d}(\pi)$ acts on $\mathcal{S}(M)$ via Wall-realisation and also that the induced homomorphism $\lambda(M)_{*}: \pi \rightarrow G$ together with the $G$-signature define a homomorphism $\sigma_{\lambda(M)}: L_{2 d}(\pi) \rightarrow \mathbb{Q} R_{\widehat{G}}^{(-1)^{d}}$. It is well known that this action is additive with respect to $\widetilde{\rho}$ (see Petrie [20]): if $x \in L_{2 d}(\pi$ ) and $[h] \in \mathcal{S}(M)$ then

$$
\widetilde{\rho}([h]+x)=\tilde{\rho}([h])+\sigma_{\lambda}(x) .
$$

Moreover, calculations of Wall [32, Chapter 14E] and the second author and Wegner [14] show that $\tilde{\rho}$ is a homomorphism when $M$ is a lens space. Wolfgang Lück asked whether this is true in general and a positive answer to this question is our main theorem.

Theorem 1.1 Let $M$ be a closed oriented topological manifold of dimension $2 d-1 \geq 5$ with a reference map $\lambda(M): M \rightarrow B G$ where $G$ is a finite group. Then the map

$$
\widetilde{\rho}: \mathcal{S}(M) \longrightarrow \mathbb{Q} R_{\widehat{G}}^{(-1)^{d}}
$$

is a homomorphism of abelian groups.

We see that Theorem 1.1 is a generalisation of the long standing identity (1-2). One may also take the point of view that it sheds light on the group structure on $\mathcal{S}(M)$. Clearly it has the potential to aid in computations of $\mathcal{S}(M)$ and this is shown to be the case in a forthcoming paper of Davis and Lück [7] about torus bundles over lens spaces. Clearly we also have:

Corollary 1.2 The map $\tilde{\rho}$ factors through $\mathcal{S}(M) \longrightarrow \mathcal{S}(M) \otimes \mathbb{Q}$.

\footnotetext{
${ }^{1}$ Our results work equally well for the simple structure set; see Remark 1.7.
} 


\subsection{The outline of the proof of Theorem 1.1}

To describe the essential ideas of the proof, we first sketch the topological definition of the $\rho$-invariant which we use throughout the paper. Let $(M, \lambda(M))$ be as above. If $Z$ is a compact oriented $2 d$-dimensional manifold with a map $\lambda(Z): Z \rightarrow B G$, we call it an $r$-coboundary for $(M, \lambda(M))$ if $\partial(Z, \lambda(Z))=\bigsqcup_{r}(M, \lambda(M))$ is the disjoint union of $r$ copies of $(M, \lambda(M))$ for some $r \geq 1$. From bordism theory we know that $r-$ coboundaries always exist for some $r$. The $G$-signature of the induced $G$-covering $\widetilde{Z}$ is an element in the complex representation ring $R(G)$. By the Atiyah-Singer $G$-index theorem [2] (see also Wall [32, Chapter 14B]), the expression

$$
\rho(M, \lambda(M)):=(1 / r) \cdot G-\operatorname{sign}(\tilde{Z})
$$

becomes independent of the choice of $Z$ and $r \geq 1$ after passing to a certain subquotient of the rationalisation of $R(G)$ (see Definition 2.2 for a precise statement).

Suppose now that we have structures $h_{0}: N_{0} \rightarrow M$ and $h_{1}: N_{1} \rightarrow M$ representing two elements in $\mathcal{S}(M)$. Unless $\left[h_{1}\right]=[\mathrm{id}]+x$ for some $x \in L_{2 d}(\pi)$, a geometric description of the structure $\left[h_{0}\right]+\left[h_{1}\right]$ in terms of $\left[h_{0}\right]$ and $\left[h_{1}\right]$ is not known at present. Thus it is not a-priori clear how to relate $r$-coboundaries for $N_{0}$ and $N_{1}$ and one sees that the additivity of the function $\tilde{\rho}$ from Equation (1-1) is not obvious.

On the other hand, the situation becomes much simpler if we replace the closed manifold $M$ by $M \times D^{l}$ for some $l \geq 1$ as we now describe. The rel boundary structure set of $M \times D^{l}, \mathcal{S}_{\partial}\left(M \times D^{l}\right)$, consists of equivalence classes of homotopy equivalences of manifolds with boundary $h:(N, \partial) \rightarrow\left(M \times D^{l}, \partial\right)$, such that the restriction to the boundary is a homeomorphism $\partial h: \partial N \cong M \times S^{l-1}$. The equivalence relation is given by $h$-cobordism of pairs in the source where the $h$-cobordism over $\partial N$ is trivial. For $l \geq 1$ there is a geometrically defined group structure using "stacking" which is easy to understand; see Definition 2.4. Suppose $n+l=\operatorname{dim}(M)+l=2 d-1$. Then following Madsen and Rothenberg [16], we define the rel boundary reduced $\rho$-invariant

(1-3) $\tilde{\rho}_{\partial}: \mathcal{S}_{\partial}\left(M \times D^{l}\right) \longrightarrow \mathbb{Q} R_{\widehat{G}}^{(-1)^{d}}, \quad\left[h: N \rightarrow M \times D^{l}\right] \longmapsto \rho\left(N \cup_{\partial h}\left(M \times D^{l}\right)\right)$.

The reference maps are left out of the notation. Notice that $N \cup_{\partial h}\left(M \times D^{l}\right)$ is a closed oriented $(2 d-1)$-dimensional manifold and so the formula makes sense. Using a certain generalised connected sum operation we prove:

Proposition 1.3 Let $M$ be a closed oriented topological manifold of dimension $n$ with a reference map $\lambda(M): M \rightarrow B G$ for a finite group $G$, and let $n+l=2 d-1 \geq 5$. Then the map

$$
\tilde{\rho}_{\partial}: \mathcal{S}_{\partial}\left(M \times D^{l}\right) \longrightarrow \mathbb{Q} R_{\widehat{G}}^{(-1)^{d}}
$$

is a homomorphism of abelian groups. 
For reasons that will become apparent later we choose $l=4 j$, and contemplate the following diagram.

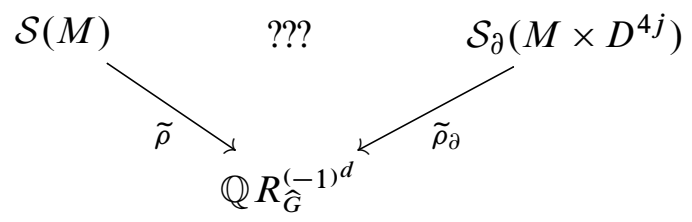

If we can find a homomorphism $\mathcal{S}(M) \rightarrow \mathcal{S}_{\partial}\left(M \times D^{4 j}\right)$ making the above diagram commute then $\tilde{\rho}$ is a homomorphism and we are done. This brings us to periodicity in topological surgery which we discuss in more detail in Section 1.2 below. For now we simply note that there is an injective near periodicity map $P^{j}: \mathcal{S}(M) \rightarrow \mathcal{S}_{\partial}\left(M \times D^{4 j}\right)$ defined by Siebenmann [31] and in a different way by Ranicki [23; 26]. However both definitions are complicated and require one to travel a long journey away from the geometry of a structure $[h: N \rightarrow M] \in \mathcal{S}(M)$. The distance is large enough that we lose sight of $r$-coboundaries and so of the $\rho$-invariant.

A geometric passage from $\mathcal{S}(M)$ to $\mathcal{S}_{\partial}\left(M \times D^{4 j}\right)$ remained unclear until [4] where Cappell and Weinberger sketched maps $\mathrm{CW}^{j}: \mathcal{S}(M) \rightarrow \mathcal{S}_{\partial}\left(M \times D^{4 j}\right)$ for $j=1,2$ or 4 . However, their construction was given using piecewise linear techniques and so strictly applies only when all manifolds involved are triangulable, although the authors hinted at the generalisations needed for the topological case. They claimed that $\mathrm{CW}^{j}=P^{j}$ but their proof uses Sullivan's Characteristic Variety Theorem which was never published in sufficient generality. Later, Hutt tried to address these issues [11]. He gave a construction of the map $\mathrm{CW}^{1}$ for topological manifolds. However Hutt's proof of near 4-periodicity uses his own theory of Poincaré sheaves which was never published.

Much of the work in this paper goes into giving a detailed proof that the Hutt construction adapted to the map $\mathrm{CW}^{2}$ indeed realises the near periodicity map $P^{2}$. In particular we replace Hutt's use of Poincaré sheaves with algebraic surgery from [26] and thereby prove:

Theorem 1.4 Let $M$ be a closed topological manifold of dimension $n \geq 5$. The Hutt description of the Cappell-Weinberger map gives an exact sequence of homomorphisms of abelian groups:

$$
0 \longrightarrow \mathcal{S}(M) \stackrel{\mathrm{CW}^{2}}{\longrightarrow} \mathcal{S}_{\partial}\left(M \times D^{8}\right) \longrightarrow H_{0}(M ; \mathbb{Z}) .
$$

The details of the Hutt construction of the map $\mathrm{CW}^{j}$ allow us to do the following: given $(Z, \lambda(Z))$, an $r$-coboundary for $N$, the domain of a structure $[h: N \rightarrow M] \in \mathcal{S}(M)$, 
we can construct an $r$-coboundary for the domain of $\mathrm{CW}^{j}([h])$. This then allows us to prove:

Theorem 1.5 Let $M$ be a closed oriented topological manifold of dimension $(2 d-1) \geq 5$ with a reference map $\lambda: M \rightarrow B G$ for a finite group $G$. Then the following diagram commutes.

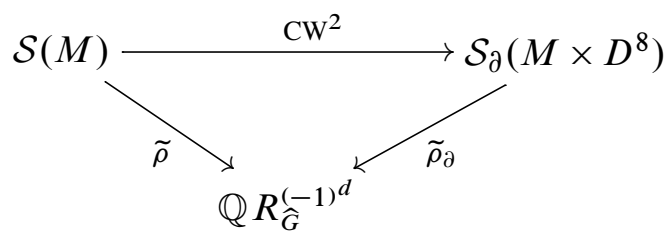

Theorem 1.1 now follows immediately from Proposition 1.3 and Theorem 1.4 and Theorem 1.5 since together they show that $\tilde{\rho}=\tilde{\rho}_{\partial} \circ \mathrm{CW}^{2}$ is a composition of homomorphisms.

Remark 1.6 The idea of understanding the group structure on $\mathcal{S}(M)$ via the stacking group structure on $\mathcal{S}_{\partial}\left(M \times D^{4 j}\right)$ and periodicity is very natural. For example in [12] Jahren and Kwasik used this method to solve an extension problem for $\mathcal{S}\left(S^{1} \times \mathbb{R} P^{n}\right)$ related to the Browder-Livesay invariant, a close cousin of the $\rho$-invariant.

\subsection{Periodicity in topological surgery}

In this subsection we briefly recall the history of periodicity in topological surgery as well as describing how this paper adds to the detailed proof of near periodicity. Let $M$ be a closed topological manifold of dimension $n \geq 5$. The source of periodicity in topological surgery is the 4-fold periodicity of the homotopy groups $\pi_{i}(G / \mathrm{TOP}) \cong$ $\pi_{i+4}(G / \mathrm{TOP})$ for $i \geq 1$. However, it took Quinn's theory of surgery spaces [21] to see how this periodicity could be extended to the structure set. Once the surgery exact sequence was identified as the long exact homotopy sequence of a fibration, Siebenmann [31] could define injective maps $P^{j}: \mathcal{S}(M) \rightarrow \mathcal{S}_{\partial}\left(M \times D^{4 j}\right)^{2}$. He used these maps to define an abelian group structure on $\mathcal{S}(M)$.

Ranicki $[23 ; 26]$ produced algebraic versions of surgery theory which translate Quinn's theory into a category of chain complexes. In particular bijections

$$
s: \mathcal{S}_{\partial}\left(M \times D^{l}\right) \rightarrow \mathbb{S}_{n+l+1}(M)
$$

${ }^{2}$ In fact Siebenmann mistakenly claimed that $P^{j}$ is a bijection. $\operatorname{In}$ general $\operatorname{Im}\left(P^{j}\right)$ is a subgroup with $\mathcal{S}\left(M \times D^{4 j}\right) / \operatorname{Im}\left(P^{j}\right)$ isomorphic to a subgroup of $H_{0}(M ; \mathbb{Z})$. Therefore to be precise we speak of near-periodicity. A correct statement of near periodicity appeared in Nicas [18]. 
are defined where $\mathbb{S}_{n+l+1}(M)$ is an abelian group. Moreover, with respect to the stacking group structure on $\mathcal{S}_{\partial}\left(M \times D^{l}\right)$ this map is an isomorphism if $l \geq 1$. Since the algebraic groups are nearly 4 -periodic almost by definition, Ranicki was able to give an algebraic proof of Siebenmann's periodicity theorem. In particular the algebraic theory of surgery so closely mirrors surgery spaces that the two group structures defined on $\mathcal{S}(M)$ agree.

As we have seen, for certain purposes the abstract descriptions of the maps $P^{j}$ do not suffice and the papers of Cappell and Weinberger [4] and Hutt [11] were written to fill this gap. For reasons mentioned above, however, neither of these papers gives a water tight proof that the maps $\mathrm{CW}^{j}: \mathcal{S}(M) \rightarrow \mathcal{S}_{\partial}\left(M \times D^{4 j}\right)$ realise the periodicity maps $P^{j}$. In the end, to give a detailed proof that $\mathrm{CW}^{2}=P^{2}$ we have had to combine important ideas from both papers and add some of our own.

For the outline of the proof of periodicity we were able to follow [11]. However to Hutt's arguments one must add foundational results of Hughes, Taylor and Williams [10] and a folk theorem proved by Hughes [9] about mapping cylinder neighbourhoods, MCNs, and manifold approximate fibrations, MAFs. We summarise these results in Theorem 3.1 and Corollary 3.3 and use them to show that Hutt's map is defined. Then one has to take more care than Hutt to show that the map is well-defined. To show that the now well-defined map $\mathrm{CW}^{j}$ is indeed $P^{j}$ we use algebraic surgery which requires an inductive dissection of a topological manifold similar to, but not in general the same as, a simplicial decomposition. In particular algebraic surgery requires that we apply Hutt's construction inductively to each space in such a dissection. Then one discovers that Theorem 3.1 concerning MCNs and MAFs has dimension restrictions which can only be satisfied for 8-periodicity. Thus we show that $\mathrm{CW}^{2}=P^{2}$ and this is sufficient to prove the additivity of the reduced $\rho$-invariant. We hope that the work in this paper might serve as a foundation to at last give a detailed proof that $\mathrm{CW}^{1}=P^{1}$.

Remark 1.7 All the results of the present paper work equally well for structure sets and simple structure sets. Thus in the familiar notation, the reader may substitute $\mathcal{S}^{s}(M)$ or $\mathcal{S}^{h}(M)$ for $\mathcal{S}(M)$ and its variants throughout the paper. To justify this we note that the $\rho$-invariant is an $h$-cobordism invariant and hence defines a function of both versions of the structure set. Moreover the forgetful map

$$
\mathcal{S}^{s}(M) \rightarrow \mathcal{S}^{h}(M)
$$

is a homomorphism. In particular the results of Quinn [21], Siebenmann [31] and Ranicki $[23 ; 26]$ work equally well for each torsion decoration. With regard to the periodicity maps $\mathrm{CW}^{j}$ our treatment is also simultaneous for both decorations: we use $h$-cobordisms throughout, but the arguments are verbally the same with $s$-cobordisms. 
In this direction our work generalises Cappell and Weinberger [4] and Hutt [11] who only deal with the $s$-decoration.

The rest of the paper is organised as follows. In Section 2 we define the $\rho$-invariant and its reduced variations. We also recall the group structure on $\mathcal{S}_{\partial}\left(M \times D^{l}\right)$ for $l \geq 1$ and we prove Proposition 1.3. The proof of Theorem 1.4 occupies Sections 3-6. In the preparatory Section 3 we recall and reformulate essential facts about MCNs and MAFs. In Section 4 we review Hutt's account of the construction of the Cappell-Weinberger map. In Section 5 we review the framework of the algebraic theory of surgery from [26] which is the key tool in the proof of Theorem 1.4 in Section 6. The proof of Theorem 1.5 occupies the last two sections. Section 7 is again preparatory and the proof is completed in Section 8.

Acknowledgements We would like to thank Jim Davis and Andrew Ranicki for helpful discussions, Bruce Hughes for enlightening correspondence at a critical juncture and Wolfgang Lück for raising the motivating question of the paper as well as helpful discussions. We would also like to thank the referee for helping to clarify a number of our proofs.

The second author was supported by SFB 478 Geometrische Strukturen in der Mathematik, Münster.

\section{The $\rho$-invariant}

Let $M$ be a closed oriented topological manifold of dimension $n=2 d-1 \geq 5$ with a reference map $\lambda(M): M \rightarrow B G$ where $G$ is a finite group. In this section we recall the definition of the reduced $\rho$-invariant function, denoted $\tilde{\rho}$, defined on the structure set $\mathcal{S}(M)$ as well as a relative $\rho$-invariant, denoted $\tilde{\rho}_{\partial}$, which is defined on the rel boundary structure set $\mathcal{S}_{\partial}\left(M \times D^{2 j}\right)$. The main outcome of the section is the proof of Proposition 1.3 which states that $\tilde{\rho}_{\partial}$ is a homomorphism.

\subsection{The $\rho$-invariant}

The $\rho$-invariant is an invariant of odd-dimensional oriented manifolds associated to the $G$-signature of cobounding even-dimensional oriented manifolds. We first briefly recall the $G$-signature. 
$G$-Signature Let $G$ be a finite group acting smoothly on a smooth oriented manifold $Z^{2 d}$. The complex intersection form $\lambda_{Z}$ on $H:=H_{d}(Z ; \mathbb{C})$ is a nondegenerate $(-1)^{d}$-symmetric bilinear form on which $G$ also acts. Choosing an inner product on $H$ expresses $\lambda_{Z}$ as a nonsingular $(-1)^{d}$-symmetric matrix and hence $H$ decomposes as $H=H_{+} \oplus H_{-}$where $H_{\varepsilon}$ is the sum of eigenspaces whose eigenvalues are positive multiples of $\varepsilon= \pm 1$ if $d$ is even and $\varepsilon i$ if $d$ is odd.

The subspaces $H_{+}$and $H_{-}$are $G$-invariant and the $G$-signature is defined to be the following virtual representation in the complex representation ring of $G$ :

$$
G-\operatorname{sign}(Z):=\left[H_{+}\right]-\left[H_{-}\right] \in R_{\mathbb{C}}(G) .
$$

Complex conjugation induces an involution on $R_{\mathbb{C}}(G)$ with $( \pm 1)$-eigenspaces. In terms of characters the $(+1)$-eigenspace corresponds to real characters and the $(-1)-$ eigenspace corresponds to purely imaginary characters. We will denote

$$
R_{\mathbb{C}}^{ \pm}(G):=\left\{\chi \pm \chi^{-1} \mid \chi \in R_{\mathbb{C}}(G)\right\} .
$$

One can also show that $G-\operatorname{sign}(Z) \in R^{(-1)^{d}}(G)$ which in terms of characters means that we obtain a real (purely imaginary) character, which will be denoted as the assignment $G-\operatorname{sign}(-, Z): g \in G \mapsto G-\operatorname{sign}(g, Z) \in \mathbb{C}$. The cohomological version of the Atiyah-Singer $G$-index theorem [2, Theorem 6.12] tells us that if $Z$ is closed then for all $g \in G$

$$
G-\operatorname{sign}(g, Z)=L(g, Z) \in \mathbb{C},
$$

where $L(g, Z)$ is an expression obtained by evaluating certain cohomological classes on the fundamental classes of the $g$-fixed point submanifolds $Z^{g}$ of $Z$. In particular if the action is free then $G-\operatorname{sign}(g, Z)=0$ if $g \neq 1$. This means that $G-\operatorname{sign}(Z)$ is a multiple of the regular representation. This theorem was generalised by Wall to topological semifree actions on topological manifolds, which is the case we will need in this paper [32, Chapter 14B]. The assumption that $Z$ is closed is essential here, and motivates the definition of the $\rho$-invariant.

Bordism groups To define the $\rho$-invariant one also needs the following result which starts with the work of Conner and Floyd [6] on smooth bordism, proceeds through Williamson [33] for piecewise linear bordism and finishes with Madsen and Milgram [15] for topological bordism.

Theorem 2.1 Let $G$ be a finite group with classifying space $B G$ and let $\Omega_{n}^{\mathrm{STOP}}(B G)$ denote bordism group of $n$-dimensional closed oriented topological manifolds with a reference map to $B G$. Then for $2 d-1 \geq 1$,

$$
\Omega_{2 d-1}^{\mathrm{STOP}}(B G) \otimes \mathbb{Q}=0 .
$$


Let $N$ be a closed oriented $(2 d-1)$-dimensional manifold with a reference map $\lambda(N): N \rightarrow B G$ inducing a homomorphism $\lambda(N)_{*}: \pi_{1}(N) \rightarrow G$. The above result means that there exists a $2 d$-dimensional oriented manifold with boundary $Z$ with a reference map $\lambda(Z): Z \rightarrow B G$ inducing a homomorphism $\lambda(Z)_{*}: \pi_{1}(Z) \rightarrow G$ such that $\partial Z=r \cdot N$ for some $r \geq 1$ and such that the restriction $\left.\lambda(Z)\right|_{\partial Z}=r \cdot \lambda(N)$. Then we also have the induced $G$-covering $\tilde{Z}$ on which the group $G$ acts freely via deck transformations. It is a manifold with boundary $r \cdot \tilde{Y}, r$ copies of the induced $G$-covering of $Y$. The above considerations make it possible to make the following definition.

Definition 2.2 [2, Section 7] Let $N$ be a closed oriented topological $(2 d-1)-$ dimensional manifold with a reference map $\lambda(N): N \rightarrow B G$ where $G$ is a finite group. Define

$$
\rho(N, \lambda(N)):=\frac{1}{r} \cdot G-\operatorname{sign}(\tilde{Z}) \in \mathbb{Q} R^{(-1)^{d}}(G) /\langle\mathrm{reg}\rangle=: \mathbb{Q} R_{\widehat{G}}^{(-1)^{d}}
$$

for some $r \in \mathbb{N}$ and $(Z, \partial Z)$ such that $\partial Z=r \cdot N$ and there is $\lambda(Z): Z \rightarrow B G$ restricting to $r \cdot \lambda(N)$ on $\partial Z$. The symbol $\langle\mathrm{reg}\rangle$ denotes the ideal generated by the regular representation, the symbol $\mathbb{Q} R^{ \pm}(G)$ means $\mathbb{Q} \otimes R^{ \pm}(G)$.

The $\rho$-invariant is well defined by the Atiyah-Singer $G$-index theorem [2, Theorem (6.12)] and its topological generalisation [32, Chapter 14B]. When the reference map is clear we will often leave out the map $\lambda(N)$ from the notation and simply write $\rho(N)$.

\subsection{Structure sets}

The structure set of a compact topological manifold is the basic object of study in surgery theory. When calculated it gives us understanding of the manifolds in the homotopy type of that given manifold, as shown for example in [32, Part 3].

Definition 2.3 Let $M$ be a compact $n$-dimensional manifold with boundary $\partial M$ (which may be empty). A (simple) manifold structure on $M$ relative to $\partial M$ consists of a (simple) homotopy equivalence of $n$-dimensional compact manifold with boundary

$$
(h, \partial h):(N, \partial N) \rightarrow(M, \partial M)
$$

such that $\partial h$ is a homeomorphism. Two such structures $\left(h_{1}, \partial h_{1}\right)$ and $\left(h_{2}, \partial h_{2}\right)$ are equivalent if there exists a (simple) homotopy equivalence of $(n+1)$-dimensional manifold 4-ads $H:\left(W, N_{1}, N_{2}, W_{\partial}\right) \rightarrow(M \times I, M \times\{0\}, M \times\{1\}, \partial M \times I)$, such that $\left.H\right|_{N_{1}}=h_{1}$ and $\left.H\right|_{N_{2}}=h_{2}$ and $\left.H\right|_{W_{\partial}}$ are homeomorphisms.

The (simple) structure set $\mathcal{S}_{\partial}(M)$ is defined as the set of equivalence classes of (simple) manifold structures on $M$ relative to $\partial M$. In the case where $\partial M$ is empty we write $\mathcal{S}(M)$. 
More generally, assume that $\left(M, \partial_{1} M, \partial_{2} M\right)$ is a manifold 3-ad (see [32, Chapter 0], note that one or both of $\partial_{i} M$ may be empty). A (simple) manifold structure on $M$ relative to $\partial_{1} M$ consists of a (simple) homotopy equivalence of $n$-dimensional compact manifold 3-ads

$$
\left(h, \partial_{1} h, \partial_{2} h\right):\left(N, \partial_{1} N, \partial_{2} N\right) \rightarrow\left(M, \partial_{1} M, \partial_{2} M\right)
$$

such that $\partial_{1} h$ is a homeomorphism. So one allows more flexibility on the part of the boundary $\partial_{2} M$. There is a corresponding equivalence relation which allows to one define the (simple) structure set in this setting, which is denoted $\mathcal{S}_{\partial_{1} M}(M)$. Hence $\partial_{2} M$ does not appear in the notation, which usually does not cause a confusion.

Also note that the $s$-cobordism theorem entails that, if $\operatorname{dim}(M)=n \geq 5$, then two simple manifold structures $h_{1}$ and $h_{2}$ are equivalent if and only if there exists a homeomorphism $f: N_{1} \rightarrow N_{2}$ such that $h_{2} \circ f \simeq h_{1}$ rel $\partial$.

All the results of the present paper work equally well for structure sets and simple structure sets. Thus in the familiar notation, the reader may substitute $\mathcal{S}^{s}(M)$ or $\mathcal{S}^{h}(M)$ for $\mathcal{S}(M)$ and its variants throughout the paper. To keep the language simple we will work with structure sets and manifold structures.

The main tool for determining $\mathcal{S}_{\partial}(M)$ for a specific manifold $M$, with $\operatorname{dim}(M)=$ $n \geq 5$, is the surgery exact sequence (see Wall [32, Chapter 10] and Kirby and Siebenmann [13] for definitions and details)

$$
\cdots \rightarrow L_{n+1}\left(\mathbb{Z}\left[\pi_{1}(M)\right]\right) \rightarrow \mathcal{S}_{\partial}(M) \rightarrow[M / \partial M ; \mathrm{G} / \mathrm{TOP}] \rightarrow L_{n}\left(\mathbb{Z}\left[\pi_{1}(M)\right]\right) .
$$

We remark that the expression "exact sequence" makes sense, as explained in [32, Chapter 10], despite the fact that the structure set, as defined, is only a pointed set with the base point the identity id: $(M, \partial M) \rightarrow(M, \partial M)$. On the other hand, as pointed out in the introduction, one can endow $\mathcal{S}_{\partial}(M)$ with the structure of an abelian group, which is natural in the sense that the above sequence indeed becomes an exact sequence of abelian groups. This follows from the identification of the surgery exact sequence (2-3) with the algebraic surgery exact sequence (5-6) which will be discussed in detail in Section 5.

In this section we only want to discuss the case when the compact manifold in question is of the form $M \times D^{k}$, for $M$ closed with $k \geq 1$. Then there is an easy geometric way of defining the structure of a group on $\mathcal{S}_{\partial}\left(M \times D^{k}\right)$ which is abelian if $k \geq 2$. Abstractly, this follows from the observation that $\mathcal{S}_{\partial}\left(M \times D^{k}\right)$ is the $k$-th homotopy group of a certain space, as explained for example in [32, Chapter 17A]. But we also need an explicit description of the addition. For this denote

$$
S_{ \pm}^{k-1}:=\left\{x=\left(x_{1}, \ldots, x_{k}\right) \in S^{k-1} \mid \pm x_{1} \geq 0\right\}
$$


and note that each element in $\mathcal{S}_{\partial}\left(M \times D^{k}\right)$ can be represented as a homotopy equivalence of manifold triads

$$
\left(h, \partial_{+} h, \partial_{-} h\right):\left(N, \partial_{+} N, \partial_{-} N\right) \rightarrow\left(M \times D^{k}, M \times S_{+}^{k-1}, M \times S_{-}^{k-1}\right)
$$

where $\partial_{ \pm} h$ are homeomorphisms. Further denote

$$
D_{ \pm}^{k}:=\left\{x=\left(x_{1}, \ldots, x_{k}\right) \in D^{k} \mid \pm x_{1} \geq 0\right\}
$$

and choose suitable homeomorphisms $\left(D^{k}, S_{+}^{k-1}, S_{-}^{k-1}\right) \cong\left(D_{+}^{k}, S_{+}^{k-1}, D^{k-1}\right)$ and $\left(D^{k}, S_{+}^{k-1}, S_{-}^{k-1}\right) \cong\left(D_{-}^{k}, D^{k-1}, S_{-}^{k-1}\right)$. Also note $D^{k}=D_{+}^{k} \cup_{D^{k-1}} D_{-}^{k}$.

Definition 2.4 Let $h_{i}: N_{i} \rightarrow M \times D^{k}$ with $i=1,2$ be maps which represent elements in $\mathcal{S}_{\partial}\left(M \times D^{k}\right)$. Define $h_{1}+h_{2}=h$ by

$$
h=h_{1} \cup h_{2}: N=N_{1} \cup_{g} N_{2} \rightarrow M \times D^{k}=M \times D_{+}^{k} \cup M \times D_{-}^{k}
$$

where $g: \partial_{+} N_{1} \rightarrow \partial_{-} N_{2}$ is given by $g=\left(\partial_{-} h_{2}\right)^{-1} \circ \partial_{+} h_{1}$.

\subsection{Structure sets and the $\rho$-invariant}

Next we define the reduced $\rho$-invariant functions.

Definition 2.5 Let $M$ be a closed oriented manifold of dimension $n=(2 d-1) \geq 5$ with a reference map $\lambda(M): M \rightarrow B G$ where $G$ is a finite group. Define the function

$$
\tilde{\rho}: \mathcal{S}(M) \rightarrow \mathbb{Q} R_{\widehat{G}}^{(-1)^{d}} \text { by } \quad \tilde{\rho}([h])=\rho(N, \lambda(M) \circ h)-\rho(M, \lambda(M)),
$$

where the orientation on $N$ is chosen so that the homotopy equivalence $h: N \rightarrow M$ is a map of degree 1 .

Since the $\rho$-invariant is an $h$-cobordism invariant [2, Corollary 7.5], the function $\tilde{\rho}$ is well-defined.

The definition in the relative setting comes from [16, Section 3]. We need a little preparation. Consider an element $[h]$ in $\mathcal{S}_{\partial}\left(M \times D^{k}\right)$. Let $M(h)$ be a closed manifold given by

$$
M(h):=N \cup_{\partial h}\left(M \times D^{k}\right) .
$$

If $h$ is the identity we obtain $M($ id $) \cong M \times S^{k}$, in general the map $h$ induces $M(h) \simeq M \times S^{k}$, and if $k \geq 2$ then $\pi_{1}(M(h)) \cong \pi_{1}(M)$. When $M$ is oriented we equip $N$ with an orientation so that $h$ is a map of degree 1 . The orientation on the closed manifold $M(h)$ can then be chosen so that it agrees with the given orientation on $N$ and it reverses the orientation on $M \times D^{k}$. If $M$ possess a reference map $\lambda(M): M \rightarrow B G$ then we obtain a reference map $\lambda(M(h)): M(h) \simeq M \times S^{k} \rightarrow M \rightarrow B G$. 
Definition 2.6 Let $M$ be a closed oriented manifold of dimension $n$ with a reference map $\lambda(M): M \rightarrow B G$ where $G$ is a finite group and let $k \geq 1$ be such that $n+k=$ $2 d-1 \geq 5$. Define the function

$$
\tilde{\rho}_{\partial}: \mathcal{S}_{\partial}\left(M \times D^{k}\right) \rightarrow \mathbb{Q} R_{\widehat{G}}^{(-1)^{d}} \text { by } \quad \tilde{\rho}_{\partial}([h]):=\rho(M(h), \lambda(M(h))) .
$$

Again this well-defined. Also notice that if $k \geq 1$ then $\widetilde{\rho}_{\partial}([\mathrm{id}])=0$.

Now we would like to understand the behaviour of $\tilde{\rho}_{\partial}$ with respect to + defined in Equation (2-4). First a definition and then an observation.

Definition 2.7 Let $h_{i}: N_{i} \rightarrow M \times D^{k}$ with $i=1,2$ be maps which represent elements in $\mathcal{S}_{\partial}\left(M \times D^{k}\right)$. Consider $\bar{M}\left(h_{i}\right):=M\left(h_{i}\right) \backslash \operatorname{int}\left(M \times D^{k-1} \times[-\epsilon, \epsilon]\right)$, for small $\epsilon>0$, where $D^{k-1}=D_{+}^{k} \cap D_{-}^{k}$. Identify $\partial \bar{M}\left(h_{i}\right)$ with $M \times S^{k-1}$. Define the closed oriented manifold $M\left(h_{1}\right) \#_{M} M\left(h_{2}\right)$ by

$$
M\left(h_{1}\right) \#_{M} M\left(h_{2}\right):=\bar{M}\left(h_{1}\right) \cup_{\mathrm{id} \times r} \bar{M}\left(h_{2}\right)
$$

where $r$ is an orientation reversing homeomorphism of $S^{k-1}$.

Lemma 2.8 There is a homeomorphism of oriented manifolds

$$
M\left(h_{1}\right) \#_{M} M\left(h_{2}\right) \cong M\left(h_{1}+h_{2}\right) .
$$

Proof Both sides can be identified with the union

$$
N_{1} \cup_{g_{1}} M \times S^{k-1} \times I \cup_{g_{2}} N_{2}
$$

where $g_{1}=\partial h_{1}: \partial N_{1} \rightarrow M \times S^{k-1}$ and $g_{2}=r \circ \partial h_{2}: \partial N_{2} \rightarrow M \times S^{k-1}$.

From the definition $\tilde{\rho}_{\partial}([h])=\rho(M(h))$ we see that Proposition 1.3 is equivalent to the following:

Proposition 2.9 There is an equality

$$
\rho\left(M\left(h_{1}+h_{2}\right)\right)=\rho\left(M\left(h_{1}\right)\right)+\rho\left(M\left(h_{2}\right)\right) .
$$

Proof Let $Z\left(h_{1}\right)$ be such that $\partial Z\left(h_{1}\right)=k \cdot M\left(h_{1}\right)$ and let $Z\left(h_{2}\right)$ be such that $\partial Z\left(h_{2}\right)=l \cdot M\left(h_{2}\right)$. Then $\partial l \cdot Z\left(h_{1}\right)=k l \cdot M\left(h_{1}\right)$ and $\partial k Z\left(h_{2}\right)=k l \cdot M\left(h_{2}\right)$, so we can assume $k=l$. In fact we will assume $k=l=1$, which makes the notation simple, the easy generalisation is left for the reader. Using Lemma 2.8 we build a coboundary for $M\left(h_{1}+h_{2}\right)$ from the coboundaries $Z\left(h_{1}\right)$ and $Z\left(h_{2}\right)$ by the following construction. 
Note that the manifold $M\left(h_{1}\right)$, as a boundary component of $Z\left(h_{1}\right)$, has a collar. Denote by $U\left(h_{1}\right) \subset Z\left(h_{1}\right)$ the portion of that collar along $M \times D^{k-1} \times[-\epsilon, \epsilon] \subset M\left(h_{1}\right)$. Construct the manifold $\bar{Z}\left(h_{1}\right)$ by removing $U\left(h_{1}\right)$ (the interior and a suitable part of the boundary). Then the boundary of $\bar{Z}\left(h_{1}\right)$ is decomposed as

$$
\bar{M}\left(h_{1}\right) \cup \partial^{\prime} \bar{Z}\left(h_{1}\right)=\bar{M}\left(h_{1}\right) \cup\left(M \times S^{k-1}\right) \times I \cup\left(M \times D^{k-1} \times[-\epsilon, \epsilon]\right) \times\{1\} .
$$

Similarly for $h_{2}$ instead of $h_{1}$. Recall also the orientation reversing homeomorphism $r: S^{k-1} \rightarrow S^{k-1}$ which we can extend to $r: D^{k} \rightarrow D^{k}$ and identify $D^{k-1} \times[-\epsilon, \epsilon] \cong$ $D^{k}$. We define

$$
Z:=\bar{Z}\left(h_{1}\right) \cup_{\bar{r}} \bar{Z}\left(h_{2}\right)
$$

where $\bar{r}: \partial^{\prime} Z\left(h_{1}\right) \rightarrow \partial^{\prime} Z\left(h_{2}\right)$ is the homeomorphism given by

$$
\bar{r}=(\mathrm{id} \times r \times \mathrm{id}) \cup(\mathrm{id} \times r \times\{1\}) .
$$

Figure 1 depicts the situation.

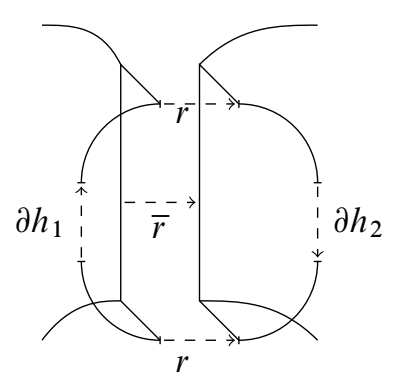

Figure 1: The coboundary $Z$

By construction the boundary of $Z$ is $M\left(h_{1}\right) \#_{M} M\left(h_{2}\right)=M\left(h_{1}+h_{2}\right)$. Then Novikov additivity gives

$$
\begin{aligned}
\rho\left(M\left(h_{1}+h_{2}\right)\right)=G-\operatorname{sign}(Z) & =G-\operatorname{sign}\left(Z\left(h_{1}\right)\right)+G-\operatorname{sign}\left(Z\left(h_{2}\right)\right) \\
& =\rho\left(M\left(h_{1}\right)\right)+\rho\left(M\left(h_{2}\right)\right) .
\end{aligned}
$$

\section{MAFs and MCNs}

This section contains preparatory material about mapping cylinder neighbourhoods which will be used in the construction of the map $\mathrm{CW}^{j}$ in the following section.

Let $X^{n} \subset Y^{n+q}, q \geq 1$, be a locally flat submanifold. A mapping cylinder neighbourhood, MCN, of $X$ is a codimension- 0 submanifold with boundary of $Y,(N, \partial N)$, such that $X \subset \operatorname{int}(N)$ and there is a deformation retraction $p: N \rightarrow X$ such that $\partial p:=\left.p\right|_{\partial N}: \partial N \rightarrow X$ satisfies $(\operatorname{cyl}(\partial p), \partial N) \cong(N, \partial N)$, where $\left.\operatorname{cyl}(\partial p)\right)$ denotes the 
mapping cylinder of $\partial p$. See Quinn [22, Section 3] for more information about the existence of MCNs.

Theorem 3.1 below, which is taken from Hughes [9], recalls a characterisation of MCNs using manifold approximate fibrations (MAFs). An approximate fibration $p: P \rightarrow N$ is a map which has an approximate homotopy lifting property. It is called a MAF if both $P$ and $N$ are manifolds. For more information we refer the reader to Hughes, Taylor and Williams [10, Section 1].

We will need the following two properties. Firstly, it follows easily from the definitions that a composition of MAFs is a MAF. Secondly, being a MAF is a local property: this means that in order to determine whether a map $p: P \rightarrow N$ between closed manifold is a MAF it is enough to check this property in a neighbourhood of each point of $N$; see [10, Corollary 12.14; 5, Proposition 2.2].

Theorem 3.1 [9, Theorem 6.1] Let $p: P \rightarrow N$ be a map between closed manifolds with $\operatorname{dim}(P)=m$ and $\operatorname{dim}(N)=n$. If $\operatorname{cyl}(p)$ is a manifold with $N$ a locally flat submanifold then $p$ is a MAF with hofib $(p) \simeq S^{m-n}$. The converse is also true if $m \geq 5$.

Remark 3.2 The above theorem is stated in [9] with the dimension restriction for both implications. However, it is clear from the proof that it is used only in one direction.

We need a generalisation of the above statement for compact manifolds with boundary. Let $(p, \partial p):(P, \partial P) \rightarrow(N, \partial N)$ be a map of compact manifolds with boundary. If $\partial N$ has more than one connected component, we index these by $\alpha \in \mathcal{I}$ and denote by $\partial_{\alpha} p: \partial_{\alpha} P \rightarrow \partial_{\alpha} N$ the corresponding restriction of $\partial p$. The mapping cylinder cyl $(\partial p)$ is a subspace of the mapping cylinder $\operatorname{cyl}(p)$ and we have

$$
\partial \operatorname{cyl}(p)=\operatorname{cyl}(\partial p) \cup_{\partial P \times\{0\}} P \times\{0\} .
$$

The triple $(\operatorname{cyl}(p) ; \operatorname{cyl}(\partial p), P \times\{0\})$ defines a triad of spaces.

Corollary 3.3 Let $(p, \partial p):(P, \partial P) \rightarrow(N, \partial N)$ be a map of compact manifolds with boundary, $\operatorname{dim}(P)=m, \operatorname{dim}(N)=n$. Assume in addition that on a collar of $\partial P$ the map $p$ is the product map of $\partial p$ with the identity in the collar direction. If the triad $(\operatorname{cyl}(p) ; \operatorname{cyl}(\partial p), P \times\{0\})$ is a manifold triad with $(N, \partial N)$ a locally flat submanifold (with boundary) of $(\operatorname{cyl}(p), \operatorname{cyl}(\partial p))$ then $(p, \partial p)$ is a pair of MAFs and we have for each connected component $\alpha \in \mathcal{I}$,

$$
\operatorname{hofib}\left(\partial_{\alpha} p\right) \simeq \operatorname{hofib}(p) \simeq S^{m-n} .
$$

The converse is also true if $m \geq 6$. 
By a pair of MAFs we just mean that both $\partial p$ and $p$ are MAFs. For a manifold triad see [32, Chapter 0].

Proof The following two observations are used. Firstly, the fact recalled above that being a MAF is a local property. Secondly, for a map $p: P^{m} \rightarrow N^{n}$ between closed manifold, we have that hofib $(p) \simeq S^{m-n}$ if and only if $(\operatorname{cyl}(p), P)$ is a Poincaré pair. Also, for $(p, \partial p):(P, \partial P) \rightarrow(N, \partial N)$, a map between compact manifolds with boundary, we have that hofib $\left(\partial_{\alpha} p\right) \simeq \operatorname{hofib}(p) \simeq S^{m-n}$ for each $\alpha \in \mathcal{I}$ if and only if $(\operatorname{cyl}(p) ; P, \operatorname{cyl}(\partial p))$ is a Poincaré triad.

The general idea is to reduce the proof to considerations about the map

$$
\bar{p}:=p \cup_{\partial p} p:\left(P \cup_{\partial P} P\right) \longrightarrow\left(N \cup_{\partial N} N\right) .
$$

To prove the if part note that $\bar{p}$ fulfils the assumptions of the if part of Theorem 3.1. It is a MAF because being a MAF is a local property and both parts are MAFs. We have hofib $(\bar{p}) \simeq S^{m-n}$, since $(\operatorname{cyl}(\bar{p}), P)$ is a Poincaré pair, because it is obtained by gluing two Poincaré triads. Hence by Theorem 3.1, $\operatorname{cyl}(\bar{p})$ is a closed manifold with $N \cup N$ locally flatly embedded. But this implies that $(\operatorname{cyl}(p), \operatorname{cyl}(\partial p) \subset \operatorname{cyl}(\bar{p})$ is a codimension -0 submanifold with boundary with $(N, \partial N)$ locally flatly embedded.

To prove the only if part observe immediately that $\operatorname{cyl}(\bar{p})$ fulfils the assumptions of Theorem 3.1. Hence $\bar{p}$ is a MAF with $\operatorname{hofib}(\bar{p}) \simeq S^{m-n}$. We obtain that $(p, \partial p)$ is a MAF pair by the locality of the MAF condition. The statement about the homotopy fibre follows immediately from the assumptions even without invoking $\bar{p}$.

Recall from surgery theory that the normal invariants of a manifold $N$, which means the bordism set of degree one normal maps with target $N$, are in one-to-one correspondence with $[N, \mathrm{G} / \mathrm{TOP}]$, homotopy classes of maps from $N$ to the space G/TOP. If instead of $N$ we consider the manifold with boundary $N \times D^{r+1}$ and if $r \geq 2$, then we have a $(\pi-\pi)-$ situation and hence

$$
\mathcal{S}\left(N \times D^{r+1}, \partial\right) \cong\left[N \times D^{r+1} ; \mathrm{G} / \mathrm{TOP}\right] \cong[N ; \mathrm{G} / \mathrm{TOP}]
$$

Therefore we can "realise" elements of $[N ; \mathrm{G} / \mathrm{TOP}]$ as homotopy equivalences of manifolds with boundary $f:(W, \partial W) \rightarrow\left(N \times D^{r+1}, \partial\right)$. As such $(W, \partial W)$ is just some manifold with boundary homotopy equivalent to $N \times D^{r+1}$. The following proposition says that there is more structure in this situation: the manifold $M$ can be identified as a MCN of $N$ and the map $f$ as the cylinder of the restriction of $f$ to the boundary. 
Proposition 3.4 Let $r \geq 2$, let $n+r \geq 5$ and let $N$ be a closed manifold of dimension $n$. For any element $\chi \in[N, \mathrm{G} / \mathrm{TOP}]$ there exists a commutative diagram

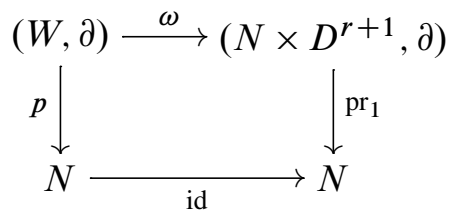

where $p: W \rightarrow N$ is a MCN with $N$ a locally flat submanifold and the map $\omega: W \rightarrow$ $N \times D^{r+1}$ is induced from a fibre homotopy equivalence of $r$-dimensional spherical fibrations associated to $p$ and $\mathrm{pr}_{1}$. In particular $\omega$ is a homotopy equivalence of pairs such that $[\omega]=\chi \in \mathcal{S}\left(N \times D^{r+1}, \partial\right) \cong[N ; \mathrm{G} / \mathrm{TOP}]$.

Proof This is proved by Hutt in [11, Section 1]. He closely follows Pedersen in [19, Proof of Lemma 9] with some additional ingredients from Quinn [22]. Pedersen's arguments are in turn based on Rourke and Sanderson [29].

In [29, Section 0] a simplicial group Top $p_{r+1}$ is defined with the following properties: (1) the set of equivalence classes of germs of codimension $(r+1)$ topological neighbourhoods of a topological manifold $N$ is in bijective correspondence with the set of homotopy classes of maps $\left[N, B \mathbf{T} o p_{r+1}\right]$, (2) there is a map $\mathbf{T} o p_{r+1} \rightarrow G_{r+1}$, the group of self-equivalences of $S^{r}$, such that the inclusions $G_{r+1} \rightarrow \mathrm{G}$ and Top $p_{r+1} \rightarrow$ TOP give rise to a homotopy equivalence $G_{r+1} /$ Top $_{r+1} \simeq \mathrm{G} / \mathrm{TOP}$.

Let $x_{r+1}: N \rightarrow G_{r+1} /$ Top $_{r+1}$ be a map representing $\chi_{r+1} \in\left[N, G_{r+1} / \mathbf{T} o p_{r+1}\right]$ where $\chi_{r+1}$ corresponds to $\chi$ under the above equivalence. If $i: G_{r+1} /$ Top $_{r+1} \rightarrow$ $B \mathbf{T} o p_{r+1}$ is the canonical map then $i \circ x_{r+1}: N \rightarrow B \mathbf{T} o p_{r+1}$ defines the germ of a codimension- $(r+1)$ topological neighbourhood of $N$. Let $V \supset N$ be such a neighbourhood. By [22, Theorem 3.1.1] there is a MCN of $N$ in $V, W \supset N$ and we let $p: \partial W \rightarrow N$ be the MAF associated to $W \supset N$. The homotopy fibre of $p$ is $S^{r}$ and we convert $p$ into a spherical fibration $S(p): S(\partial W) \rightarrow N$. So far we have only used $i \circ x_{r+1}$ : by definition the map $x_{r+1}$ defines a fibre homotopy trivialisation of $S(p)$ and so we obtain a homotopy equivalence $\tau: \partial W \simeq N \times S^{r}$. We set $\omega:=\operatorname{cyl}(\tau):(W, \partial W) \simeq\left(N \times D^{r+1}, \partial\right)$.

It remains to show that the normal invariant of $\omega$ is $\chi$. As discussed above, we have a canonical identification of the normal invariant set of $N \times D^{r+1}$ with [N, G/TOP]. The normal invariant of any degree one normal map $f:(M, \partial M) \rightarrow\left(N \times D^{r+1}, \partial\right)$ can be computed from the normal invariant of the splitting obstruction along $N \times\{0\} \subset$ $N \times D^{r+1}$. But by definition, the splitting obstruction to $\omega$ along $N \times 0$ has normal invariant $\chi$; see [15, Theorem 2.23] for this identification which was stated there for 
the $P L$-category but remains true in the topological category given the later proof of topological transversality [13; 8, Chapter 9].

We will also need a relative version of Proposition 3.4. In that case we are given a manifold with boundary $(N, \partial N)$ of dimension $n$. The product $N \times D^{r+1}$ becomes a manifold triad and we define $\partial_{0}\left(N \times D^{r+1}\right):=N \times S^{r}$ and $\partial_{1}\left(N \times D^{r+1}\right):=$ $\partial N \times D^{r+1}$. Then $\partial \partial_{1}=\partial \partial_{0}=\partial N \times S^{r}$. If $r \geq 2$ we are again in a $(\pi-\pi)$-situation and hence $\mathcal{S}\left(N \times D^{r+1}, \partial_{0}, \partial_{1}\right) \cong\left[N \times D^{r+1} ; \mathrm{G} / \mathrm{TOP}\right] \cong[N ; \mathrm{G} / \mathrm{TOP}]$.

Proposition 3.5 Let $r \geq 2, n+r \geq 5$ and let $(N, \partial N)$ be a compact manifold of dimension $n$ with boundary. Suppose given a MCN $\left(\partial_{1} W, \partial \partial_{1} W\right) \rightarrow \partial N$, with $\partial N$ a locally flat submanifold, whose associated $r$-spherical fibration is fibre homotopically trivialised by a map $\left(\partial_{1} W, \partial \partial_{1} W\right) \rightarrow\left(\partial N \times D^{r+1}, \partial N \times S^{r}\right)$ which is represented by a map $\xi: \partial N \rightarrow \mathrm{G} / \mathrm{TOP}$. For any element $\chi \in[N, \mathrm{G} / \mathrm{TOP}]$, such that $\left.\chi\right|_{\partial N}=\xi$ there exists a diagram

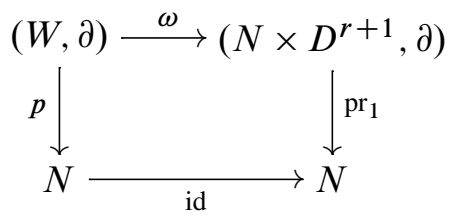

where $p: W \rightarrow N$ is a MCN with $N$ a locally flat submanifold and the map $\omega: W \rightarrow$ $N \times D^{r+1}$ is induced from a fibre homotopy equivalence of $r$-dimensional spherical fibrations associated to $p$ and $\mathrm{pr}_{1}$. In particular $\omega$ is a homotopy equivalence of pairs, and restrictions of everything to the appropriate parts of boundary agree with the given structures, such that $[\omega]=\chi$.

Proof The theorems of $[29 ; 22 ; 15]$ used in the proof of Proposition 3.4 have suitable relative versions. The proof is then a routine modification of the arguments in Proposition 3.4.

Remark 3.6 In Proposition 3.5 we had another possibility: to start with a map $N \rightarrow$ G/TOP without specifying the MCN over the boundary. We could then use the absolute version to produce such a MCN and then use the relative version. In that case, however, the dimension restrictions would have to be relaxed by 1 which would be inconvenient later.

\section{The Cappell-Weinberger map}

In this section we recall Hutt's description of the Cappell-Weinberger map and prove some basic facts about this map. We present the map both for the usual 4-periodicity 
and also for 8 -periodicity: a possibility pointed out in [4, p. 48]. Thus let $\mathbb{F}=\mathbb{C}$ or $\mathbb{H}$ and let $k=2$ or 4 be the dimension of $\mathbb{F}$ over $\mathbb{R}$.

Let $h: N \rightarrow M$ be a homotopy equivalence of closed topological manifolds of dimension $n \geq 5$ representing $[h] \in \mathcal{S}(M)$. From $h$ the Hutt construction produces a homotopy equivalence $h^{\prime}: N^{\prime} \rightarrow M \times D^{2 k}$ of manifolds with boundary defined by (4-4) and (4-5) below. The restriction of $h^{\prime}$ to the boundary of $N^{\prime}$ is a homeomorphism and so $h^{\prime}$ represents an element $\left[h^{\prime}\right] \in \mathcal{S}_{\partial}\left(M \times D^{2 k}\right)$. The mapping of structures, $[h] \mapsto\left[h^{\prime}\right]$, is the CW-map of Definition 4.2. The rest of the first subsection is devoted to proving that this map is well defined. In the second subsection we will review the construction of a homotopy equivalence of closed manifolds $\hat{h}: \hat{N} \rightarrow M \times \mathbb{F} P^{2}$ given by "extension by a homeomorphism" from $h^{\prime}$. In Lemma 4.9 we will show that this structure is equivalent in $\mathcal{S}\left(M \times \mathbb{F} P^{2}\right)$ to another structure, $\bar{h}: \bar{N} \rightarrow M \times \mathbb{F} P^{2}$ which has a certain factorisation, whereas $h^{\prime}$ does not possess an analogous factorisation.

It is not immediately clear that the Hutt construction produces a periodicity map. We prove this later in Section 6 for $\mathbb{F}=\mathbb{H}$. We note that an essential component of the construction is the use of certain $S^{k-1}$-branched coverings. This permits the extension by a homeomorphism mentioned above, which is a key ingredient in the proof of the fact that the CW-map is a periodicity map.

It is useful to observe that the constructions of $h^{\prime}, \hat{h}$ and $\bar{h}$ have two components. It is one thing to construct the sources $N^{\prime}, \widehat{N}$ and $\bar{N}$ of the above maps and it is another issue to construct the maps to $M \times D^{2 k}$ and $M \times \mathbb{F} P^{2}$. In particular it is easier to construct the sources. We use this point in the last Section 8 , where we apply a version of the Hutt construction for manifolds with boundary to construct a coboundary for the closed manifold $\bar{N}$.

\subsection{Definition}

Let $h: N \rightarrow M$ be a homotopy equivalence of closed topological manifolds of dimension $n \geq 5$ representing $[h] \in \mathcal{S}(M)$. Our starting point is the following:

Lemma 4.1 [11, Section 1] There is a commutative diagram of maps of pairs

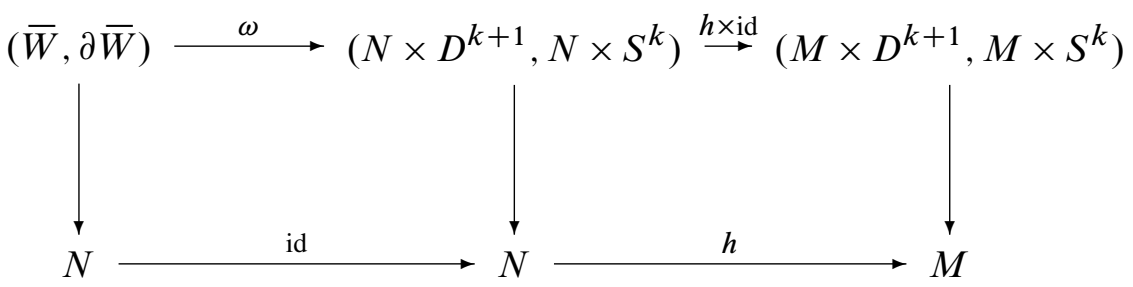


where $\bar{W}$ is a mapping cylinder neighbourhood of $N$ in which $N$ is locally flatly embedded and $\omega: \bar{W} \rightarrow N \times D^{k+1}$ is a homotopy equivalence of pairs such that the composite $\bar{\psi}:=(h \times \mathrm{id}) \circ \omega$ is $h$-cobordant, as a map of pairs, to a homeomorphism.

Proof The homotopy equivalence $h \times$ id defines an element of $\mathcal{S}\left(M \times D^{k+1}\right)$ which, by the $(\pi-\pi)$-theorem [32, Chapter 4], is isomorphic to the normal invariant set $\mathcal{N}\left(M \times D^{k+1}\right) \cong[M, \mathrm{G} / \mathrm{TOP}]$. Hence $h \times$ id defines an element $[h \times \mathrm{id}] \in[M, \mathrm{G} / \mathrm{TOP}]$. We choose $\chi \in[N, \mathrm{G} / \mathrm{TOP}]$ so that $\left(h^{-1} \times \mathrm{id}\right)^{*}(\chi)=-[h \times \mathrm{id}]:$ here we take the negative with the Whitney sum group structure on G/TOP and note that since $\left(h^{-1}\right)^{*}$ induces an isomorphism of $[N, \mathrm{G} / \mathrm{TOP}] \cong[M, \mathrm{G} / \mathrm{TOP}]$, such a $\chi$ exists. By Proposition 3.4 the element $\chi \in[N, \mathrm{G} / \mathrm{TOP}]$ gives rise to a homotopy equivalence of pairs

$$
\omega:(\bar{W}, \partial \bar{W}) \rightarrow\left(N \times D^{k+1}, N \times S^{k}\right)
$$

with the claimed properties: $\bar{W}$ is a mapping cylinder neighbourhood of $N$ in which $N$ is locally flatly embedded and $\omega(N)=N \times\{0\} \subset N \times D^{k+1}$.

Finally, by the composition formula of [3, Proposition 2.2] and [17, Lemma 2.5] $(h \times$ id $) \circ \omega$ has trivial normal invariant and hence $\bar{\psi}:=(h \times$ id $) \circ \omega$ is h-cobordant to a homeomorphism as required.

Lemma 4.1 states in particular that there is a homotopy equivalence

$$
H:\left(U ; \bar{W}, W, U_{\partial}\right) \rightarrow\left(M \times D^{k+1} \times[0,1] ;\{0\},\{1\}, M \times S^{k} \times[0,1]\right)
$$

where $U$ is an $h$-cobordism of manifolds with boundary; we denote $\partial U=\bar{W} \cup W \cup U_{\partial}$, where $U_{\partial}$ is an $h$-cobordism between $\partial \bar{W}$ and $\partial W$. Also $\left.H\right|_{W}=\bar{\psi}$ and $\left.H\right|_{W}$ is a homeomorphism $\left.H\right|_{W}=: \psi:(W, \partial W) \cong\left(M \times D^{k+1}, M \times S^{k}\right)$. For later use, we write $H_{\partial}: U_{\partial} \rightarrow M \times S^{k} \times[0,1]$ for the restriction of $H$ to the boundary part $U_{\partial}$. Note that this differs from Hutt who only uses $H_{\partial}$ and calls it $H$. As Hutt observes, $\bar{\psi}$ and $\psi$ are maps with contrasting properties: $\bar{\psi}$ is a map over $h$ which is not in general a homeomorphism whereas $\psi$ is a homeomorphism but not in general a map over $h .^{3}$ We shall need to exploit these two properties in the following construction and therefore slide between them via the map $H$.

The key part of the construction is the following " $S^{k-1}$-branched cover plus $h-$ cobordism construction" of [4, Section 1]. Let $\gamma$ denote the Hopf bundle $S^{2 k-1} \rightarrow S^{k}$ and for a manifold $X$ let $\gamma_{X}:=\operatorname{id}_{X} \times \gamma: X \times S^{2 k-1} \rightarrow X \times S^{k}$. Further let $\partial \psi=\left.\psi\right|_{\partial W}$,

\footnotetext{
${ }^{3}$ In fact we do not even have a preferred map from $W$ to $N$. We could use the $h$-cobordism $U$ to obtain some map, but still the map $\psi$ would not be a map over $h$ in general.
} 
$\partial \bar{\psi}=\left.\bar{\psi}\right|_{\partial \bar{W}}$ and $\partial \omega=\left.\omega\right|_{\partial \bar{W}}$. Define

$$
\begin{aligned}
\partial \bar{W}^{\prime}:=(\partial \bar{\psi})^{*}\left(\gamma_{M}\right) & \cong(\partial \omega)^{*}\left(\gamma_{N}\right), \partial W^{\prime}:=(\partial \psi)^{*}\left(\gamma_{M}\right), \\
U_{\partial}^{\prime} & :=\left(H_{\partial}\right)^{*}\left(\gamma_{M \times[0,1]}\right)
\end{aligned}
$$

to be the pullbacks. ${ }^{4}$ Observe that the manifold $U_{\partial}^{\prime}$ is an $h$-cobordism between $\partial \bar{W}^{\prime}$ and $\partial W^{\prime}$, since $U_{\partial}$ was an $h$-cobordism between $\partial \bar{W}$ and $\partial W$. Further denote $\partial \bar{\psi}^{\prime}: \partial \bar{W}^{\prime} \rightarrow M \times S^{2 k-1}, \quad \partial \psi^{\prime}: \partial W^{\prime} \rightarrow M \times S^{2 k-1}, \quad H_{\partial}^{\prime}: U_{\partial}^{\prime} \rightarrow M \times S^{2 k-1} \times[0,1]$ the maps covering $\partial \bar{\psi}, \partial \psi$, and $H_{\partial}$ respectively. The $S^{k-1}$-bundle projections are denoted by

$$
q \bar{W}: \partial \bar{W}^{\prime} \rightarrow \partial \bar{W}, \quad q_{W}: \partial W^{\prime} \rightarrow \partial W \quad \text { and } \quad q_{U}: U_{\partial}^{\prime} \rightarrow U_{\partial} .
$$

By Theorem 3.1 the map $p: \partial \bar{W} \rightarrow N$ is a MAF over $N$ with homotopy fibre $S^{k}$. Obviously $q \bar{W}$ is also a MAF. As the composition of MAFs is a MAF, the map

$$
p^{\prime}: \partial \bar{W}^{\prime} \stackrel{q \bar{W}}{\longrightarrow} \partial \bar{W} \stackrel{p}{\rightarrow} N
$$

is a MAF with homotopy fibre $S^{2 k-1}$. Again applying Theorem 3.1 it follows that $\operatorname{cyl}\left(p^{\prime}\right)$, the mapping cylinder of $p^{\prime}$, is a $\mathrm{MCN}$ of $N$ with $N$ locally flatly embedded. Denote

$$
N^{\prime}:=\operatorname{cyl}\left(p^{\prime}\right) \cup U_{\partial}^{\prime} .
$$

The topological manifold $N^{\prime}$, with boundary $\partial W^{\prime}$, will be the domain of the structure used to define the Cappell-Weinberger map. The $S^{k-1}$-branched cover refers to the projection map $\operatorname{cyl}\left(p^{\prime}\right) \rightarrow \operatorname{cyl}(p)$ which can be viewed as such, the branching subset being $N \subset \operatorname{cyl}(p)$.

Next we define a homotopy equivalence $h^{\prime}: N^{\prime} \rightarrow M \times D^{2 k}$ whose restriction to the boundary $\left.h^{\prime}\right|_{\partial W^{\prime}}: \partial W^{\prime} \rightarrow M \times S^{2 k-1}$ is a homeomorphism. We regard $M \times D^{2 k}$ as the union $\left(M \times D_{1}^{2 k}\right) \cup_{M \times S^{2 k-1} \times\{1\}}\left(M \times S^{2 k-1} \times[1,2]\right)$ where $D_{1}^{2 k}$ has radius 1 and we reparametrise $H_{\partial}^{\prime}: U_{\partial}^{\prime} \rightarrow M \times S^{2 k-1} \times[1,2]$. Now define

$$
h^{\prime}:=\operatorname{cyl}\left(\partial \bar{\psi}^{\prime}, h\right) \cup H_{\partial}^{\prime}: N^{\prime} \rightarrow M \times D^{2 k} .
$$

We remark again that $h^{\prime}$ is not a map over $h$, since the map $\partial \psi$, and hence the maps $\partial \psi^{\prime}$ and $H_{\partial}^{\prime}$ are not maps over $h$.

Definition 4.2 For $k=2$ or 4 the Cappell-Weinberger map is the map

$$
\mathrm{CW}^{k / 2}: \mathcal{S}(M) \rightarrow \mathcal{S}_{\partial}\left(M \times D^{2 k}\right), \quad[h] \longmapsto\left[h^{\prime}\right] .
$$

\footnotetext{
${ }^{4}$ The notation should be understood as $(\partial W)^{\prime}$, not $\partial\left(W^{\prime}\right)$, in fact there is no manifold $W^{\prime}$.
} 
Remark 4.3 The above construction is in fact quite subtle. Note that the map $\bar{\psi} \cup H_{\partial}$ is $h$-cobordant rel $\partial$ to a homeomorphism, hence representing the trivial element in $\mathcal{S}_{\partial}\left(M \times D^{k+1}\right)$. On the other hand, this argument cannot be used for $h^{\prime}$. The point is that $N^{\prime}$ cannot be identified with the pull back of $M \times D^{2 k} \rightarrow M \times D^{k+1}$ along $\bar{\psi}$. If we wanted to have such an identification we would need $\bar{\psi}$ to be transverse to $M=M \times\{0\} \in M \times D^{k+1}$. And $\bar{\psi}$ is in general not transverse to this submanifold (despite the equality $\left.(\bar{\psi})^{-1}(M)=N \subset \bar{W}\right)$. If this were the case, then we would immediately obtain that $h$ is normally cobordant to a homeomorphism, which is not the case in general.

The remainder of this subsection is devoted to proving that the structure invariant $\left[h^{\prime}\right]$ is independent of all the choices made during its construction from the structure invariant $[h]$. In turn these are:

(1) the choice of $h: N \rightarrow M$ to represent $[h] \in \mathcal{S}(M)$,

(2) the choice of the homotopy equivalence $\omega:(\bar{W}, \partial \bar{W}) \rightarrow\left(N \times D^{k+1}, N \times S^{k}\right)$ representing an element in $\mathcal{S}\left(N \times D^{k+1}\right)$,

(3) the choice of the $h$-cobordism $U$, between $\bar{W}$ and some manifold $W$, with the homotopy equivalence $H: U \rightarrow M \times D^{k+1} \times[0,1]$ such that $H \mid \bar{W}=\bar{\psi}$ and $\left.H\right|_{W}=\psi$ which is some homeomorphism.

Let $(h, \omega, H)^{\prime}$ be the structure on $M \times D^{2 k}$ produced from a choice of $h, \omega$ and $H$. If two homotopy equivalences $h_{i}: N_{i} \rightarrow M, i=0,1$, represent the same element in $\mathcal{S}(M)$, then there is an $h$-cobordism $\left(Z ; N_{0}, N_{1}\right)$ and a homotopy equivalence $h_{Z}: Z \rightarrow M \times[0,1]$ with restrictions $\left.h_{Z}\right|_{N_{i}}=h_{i}$. The constructions of Lemma 4.1 can be applied in the relative setting. This means that $h_{Z}$ can be precomposed with a homotopy equivalence $\omega_{Z}: \bar{X} \rightarrow Z \times D^{k+1}$ from some $h$-cobordism $\left(\bar{X} ; \bar{W}_{0}, \bar{W}_{1}\right)$, which is also a MCN of $\left(Z ; N_{0}, N_{1}\right)$ and we obtain a homotopy equivalence

$$
\bar{\Psi}: \bar{X} \rightarrow M \times D^{k+1} \times[0,1]
$$

which restricts to $\bar{\Psi} \mid \bar{W}_{i}=\bar{\psi}_{i}$ for some homotopy equivalences $\bar{\psi}_{i}$ which are $h-$ cobordant to homeomorphisms $\psi_{i}$.

The choice of $\omega$ produces a similar outcome. For $i=0,1$, two homotopy equivalences $\omega_{i j}: \bar{W}_{i j} \rightarrow N_{i} \times D^{k+1}$, for $j=0,1$, as in Lemma 4.1 represent the same element of $\mathcal{S}\left(N_{i} \times D^{k+1}\right)$, so again there is an $h$-cobordism $\left(\bar{X}_{i}, \bar{W}_{i 0}, \bar{W}_{i 1}\right)$, which is also a MCN of $\left(N_{i} \times[0,1], N_{i} \times\{0\}, N_{i} \times\{1\}\right)$ and also a homotopy equivalence $\omega_{N_{i} \times D^{k+1}}: \bar{X}_{i} \rightarrow$ $N_{i} \times[0,1] \times D^{k+1}$. Composing with $h_{i} \times \mathrm{id}: N_{i} \times[0,1] \times D^{k+1} \rightarrow M \times[0,1] \times D^{k+1}$ we obtain $\bar{\Psi}_{i}$ with analogous properties as the map $\bar{\Psi}$ in (4-6). We can glue $\bar{X}, \bar{X}_{0}$ 
and $\bar{X}_{1}$ along their common boundary components. There is a corresponding homotopy equivalence to $M \times D^{k+1} \times[0,1]$. This has precisely the same properties as $\bar{\Psi}$ in (4-6), so we may assume from now on that $\bar{\Psi}$ represents the difference between choices $\left(h_{0}, \omega_{0}\right)$ and $\left(h_{1}, \omega_{1}\right)$.

Now we come to the choice of $H$. Consider a pair of $h$-cobordisms $U_{i}, i=0,1$, each from $\bar{W}_{i}$ to $W_{i}$, with homotopy equivalences $H_{i}$ between $\bar{\psi}_{i}$ and homeomorphisms $\psi_{i}$. We can glue these two $h$-cobordisms onto $\bar{X}$ along $\bar{W}_{0}$ and $\bar{W}_{1}$, take a product of the result with $[0,1]$ and rearrange the boundary to obtain a compact manifold $Y$ with boundary. The manifold $Y$ can be seen as an $h$-cobordism either between $h$-cobordisms $U_{0}$ and $U_{1}$ or between the $h$-cobordism $\bar{X}$ and some $h$-cobordism $\left(X ; W_{0}, W_{1}\right)$. We also obtain a homotopy equivalence

$$
G:(Y ; \bar{X}, X) \rightarrow\left(M \times D^{k+1} \times[0,1] \times[0,1],[0,1] \times\{0\},[0,1] \times\{1\}\right)
$$

restricting to $\bar{\Psi}$ on the $h$-cobordism $\left(\bar{X} ; \bar{W}_{0}, \bar{W}_{1}\right)$ and to a homotopy equivalence $\Psi: X \rightarrow M \times D^{k+1} \times[0,1]$ on $\left(X ; W_{0}, W_{1}\right)$ such that $\left.\Psi\right|_{W_{i}}=\psi_{i}$, which are homeomorphisms. Further $\left.G\right|_{U_{i}}=H_{i}$.

But we may also view $Y$ as an $h$-cobordism between $U_{0} \cup X$ and $U_{1}$ (just thicken $W_{1}$ to $W_{1} \times[0,1]$ and rearrange the boundary again). The point is that under this change of viewpoint the homotopy equivalence $G$ is a homeomorphism on $W_{1} \times[0,1]$.

Recall the constructions between Lemma 4.1 and Definition 4.2 which from the homotopy equivalence $H$ of (4-1) produce the rel $\partial$ structure $h^{\prime}$ on $M \times D^{2 k}$ of (4-5). Using Corollary 3.3 in place of Theorem 3.1 we may now perform the precisely analogous constructions with the homotopy equivalence $G$ to obtain a rel $\partial D^{2 k} \times[0,1]$ structure on $M \times D^{2 k} \times[0,1]$. This structure restricts to $\left(h_{0}, \omega_{0},\left.H_{0} \cup G\right|_{X}\right)^{\prime}$ and $\left(h_{1}, \omega_{1}, H_{1}\right)^{\prime}$ on the respective ends and hence gives an $h$-cobordism rel $\partial D^{2 k} \times[0,1]$ between these two rel $\partial D^{2 k}$ structures on $M \times D^{2 k}$.

(Here the role of $U$ in (4-1) is played by Y, that of $\bar{W}$ by $\bar{X}$, that of $W$ by $W_{1} \times[0,1]$ and that of $U_{\partial}$ by the part of the boundary of $Y$ which constitutes the $h$-cobordism between $\left(U_{0}\right)_{\partial} \cup X_{\partial}$ and $\left(U_{1}\right)_{\partial}$ where $X_{\partial}$ is the part of the boundary of $X$ which is the $h$-cobordism between $\partial W_{0}$ and $\partial W_{1}$. Keep in mind that $\left(\bar{X} ; \bar{W}_{0}, \bar{W}_{1}\right)$ is a MCN of $\left(Z ; N_{0}, N_{1}\right)$.)

Thus we have proved:

Lemma 4.4 With $G$ as above $\left[\left(h_{0}, \omega_{0},\left.H_{0} \cup G\right|_{X}\right)^{\prime}\right]=\left[\left(h_{1}, \omega_{1}, H_{1}\right)^{\prime}\right] \in \mathcal{S}_{\partial}\left(M \times D^{2 k}\right)$.

It remains to show $\left[\left(h_{0}, \omega_{0}, H_{0}\right)^{\prime}\right]=\left[\left(h_{0}, \omega_{0},\left.H_{0} \cup G\right|_{X}\right)^{\prime}\right]$ which we prove in the following paragraphs. 
Consider now $\left.G\right|_{X}: X \rightarrow M \times D^{k+1} \times[0,1]$ which is an h-cobordism between the homeomorphisms $\psi_{0}$ and $\psi_{1}$. As such $\left.G\right|_{X}$ defines an element of $\mathcal{S}_{\partial\{0,1\}}\left(M \times D^{k+1} \times[0,1]\right)$ where the subscript $\partial\{0,1\}$ indicates that all structure invariants are defined relative to $M \times D^{k+1} \times\{0,1\}$. Using the $S^{k-1}$-branched cover viewpoint we will next show that from $\left.G\right|_{X}$ we can obtain a structure from $M \times D^{2 k} \times[0,1]$ relative to $M \times D^{2 k} \times\{0,1\}$ which relates $\left(h_{0}, \omega_{0}, H_{0}\right)^{\prime}$ and $\left(h_{0}, \omega_{0},\left.H_{0} \cup G\right|_{X}\right)^{\prime}$.

The Hopf fibration $S^{2 k-1} \rightarrow S^{k}$ is given by a free $S^{k-1}$-action on $S^{2 k-1}$. If we take the cone of this action we obtain an $S^{k-1}$ action on $D^{2 k}$, free except at the centre point, which exhibits $D^{2 k}$ as a branched $S^{k-1}$-fibration over $D^{k+1}=$ $D^{2 k} / S^{k-1}$. Taking the product with $M$ we have $M \times D^{2 k} \rightarrow M \times D^{k+1}$ which is a branched $S^{k-1}$-fibration with branch set $M \times\{0\} \subset M \times D^{k+1}$. Now let $f: X \rightarrow M \times D^{k+1} \times[0,1]$ represent $[f] \in \mathcal{S}_{\partial\{0,1\}}\left(M \times D^{k+1} \times[0,1]\right)$. If we make $f$ transverse to $M \times\{0\} \times[0,1]$, then we may pull back the branched $S^{k-1}$-fibration $\Gamma: M \times D^{2 k} \times[0,1] \rightarrow M \times D^{k+1} \times[0,1]$ along $f .^{5}$ The outcome, $f^{*}(\Gamma)$, is a branched $S^{k-1}$-fibration over $X$ which defines a structure on $M \times D^{2 k} \times[0,1]$ which is relative to $M \times D^{2 k} \times\{0,1\}$. Using transversality along the $h$-cobordisms which define the equivalence relation in $\mathcal{S}_{\partial\{0,1\}}\left(M \times D^{k+1} \times[0,1]\right)$ we obtain a well defined map

$$
\Gamma^{*}: \mathcal{S}_{\partial\{0,1\}}\left(M \times D^{k+1} \times[0,1]\right) \rightarrow \mathcal{S}_{\partial\{0,1\}}\left(M \times D^{2 k} \times[0,1]\right), \quad[f] \mapsto\left[f^{*}(\Gamma)\right] .
$$

We have an obvious map

$$
R: \mathcal{S}_{\partial\{0,1\}}\left(M \times D^{2 k} \times[0,1]\right) \rightarrow \mathcal{S}_{\partial\{0,1\}}\left(M \times S^{2 k-1} \times[0,1]\right)
$$

and an obvious action

$$
\text { col: } \mathcal{S}_{\partial\{0,1\}}\left(M \times S^{2 k-1} \times[0,1]\right) \times \mathcal{S}_{\partial}\left(M \times D^{2 k}\right) \rightarrow \mathcal{S}_{\partial}\left(M \times D^{2 k}\right)
$$

given, respectively, by restricting to the boundary and by adding a collar. It is straight forward to verify that there is an identity of structures invariants

(4-7) $\left[\left(h_{0}, \omega_{0},\left.H_{0} \cup G\right|_{X}\right)^{\prime}\right]=\operatorname{col}\left(\left(R\left(\Gamma^{*}\left(\left[\left.G\right|_{X}\right]\right)\right),\left[\left(h_{0}, \omega_{0}, H_{0}\right)^{\prime}\right]\right) \in \mathcal{S}_{\partial}\left(M \times D^{2 k}\right)\right.$.

The following two general Lemmas then complete our proof that $\mathrm{CW}^{k / 2}([h])=\left[h^{\prime}\right]$ is well-defined.

Lemma 4.5 Let $M$ be a closed $n$-dimensional manifold and let $l \geq 3$ be such that $n+l \geq 5$. Then the following action is trivial:

$$
\operatorname{col} \circ(R \times \mathrm{Id}): \mathcal{S}_{\partial\{0,1\}}\left(M \times D^{l} \times[0,1]\right) \times \mathcal{S}_{\partial}\left(M \times D^{l}\right) \rightarrow \mathcal{S}_{\partial}\left(M \times D^{l}\right) .
$$

${ }^{5}$ Note that $f$ is by definition a homeomorphism on $\partial\{0,1\}$, hence transverse to anything, hence does not have to be changed on $\partial\{0,1\}$. 
Proof Represent $[G] \in \mathcal{S}_{\partial\{0,1\}}\left(M \times D^{l} \times[0,1]\right)$ by a structure $G: X \rightarrow M \times D^{l} \times[0,1]$. Let $F: N^{\prime} \rightarrow M \times D^{l}$ represent $[F] \in \mathcal{S}_{\partial}\left(M \times D^{l}\right)$. Consider extending $F$ by $\operatorname{col}(R(G))$, which can be conveniently denoted as $R(G) \cup F$. We now have two structures, $F$ and $R(G) \cup F$ on $M \times D^{l}$ and we need to show that they represent the same element of $\mathcal{S}_{\partial}\left(M \times D^{l}\right)$, that is, we need to find an $h$-cobordism between them.

One way to think about $G$ is as of a homotopy between two homeomorphisms $G_{0}$ and $G_{1}$, where $G_{i}: X_{i} \rightarrow M \times D^{l} \times\{i\}$ are the appropriate restrictions. But we can also think of $G$ as a rel boundary $h$-cobordism between $G_{1} \cup R(G)$ and $G_{0}$. This shows that $G_{1} \cup R(G)$, when thought of as an element of the structure set of $M \times D^{l}$ is trivial, since $G_{0}$ is a homeomorphism.

Now both structures $F$ and $R(G) \cup F$ on $M \times D^{l}$ can be extended by a homeomorphism to structures on $M \times S^{l}$, namely $G_{1} \cup R(G) \cup F$ and $G_{0} \cup F$. But we can now glue $G$ considered as an $h$-cobordism as above with the trivial $h$-cobordism $F \times$ id to obtain an $h$-cobordism between the two structures $G_{1} \cup R(G) \cup F$ and $G_{0} \cup F$ on $M \times S^{l}$. This means that they represent the same element in the structure set of $M \times S^{l}$. By Lemma 4.6 below the structures $R(G) \cup F$ and $F$ represent the same element of $\mathcal{S}_{\partial}\left(M \times D^{l}\right)$.

For the statement of the next lemma, recall that if $P$ if a closed manifold with a decomposition $P=Q \cup C$, where $Q$ and $C$ are codimension 0 submanifolds. Then there is a well defined a map $E: \mathcal{S}_{\partial}(Q) \rightarrow \mathcal{S}(P)$ given by extension with a homeomorphism.

Lemma 4.6 Let $M$ be a closed $n$-dimensional manifold and let $l \geq 3$ with $n+l \geq 5$. Then the extension by a homeomorphism map

$$
E: \mathcal{S}_{\partial}\left(M \times D^{l}\right) \rightarrow \mathcal{S}\left(M \times S^{l}\right)
$$

is injective.

Proof We establish some notation. Let $\pi=\pi_{1}(M)$, let $\Sigma^{j} X$ denote $j$-fold reduced suspension of a space X, let $X_{+}$denote $X$ with a disjoint basepoint, let $T=M \times S^{l}$, let $p: T \rightarrow M$ be the projection, let $i: M \rightarrow T$ be the obvious inclusion and let $c: T \rightarrow T / i(M)=\Sigma^{l} M_{+}$be the collapse map so that we have a cofibration

$$
M \stackrel{i}{\longrightarrow} T \stackrel{c}{\longrightarrow} \Sigma^{l} M_{+} .
$$

We leave the reader to verify that $E$ fits into the following commutative diagram whose rows are fragments of the topological surgery exact sequences for $M \times D^{l}$ and 


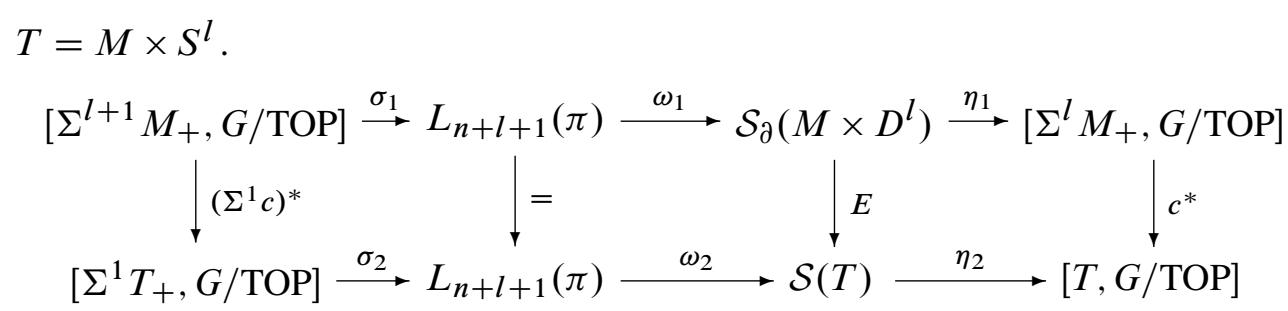

Here we have identified $\left(M \times D^{l}\right) /\left(M \times S^{l-1}\right)$ with $\Sigma^{l} M_{+}$and $c^{*}$ and $\left(\Sigma^{1} c\right)^{*}$ denote respectively precomposition with $c$ and its suspension. Note that $G /$ TOP is an infinite loop space, $c^{*}$ is split by $p^{*}$ and so $c^{*}$ and $\left(\Sigma^{1} c\right)^{*}$ are split injective.

Now let $\left[f_{1}\right],\left[f_{2}\right] \in \mathcal{S}_{\partial}\left(M \times D^{l}\right)$ be two structure invariants and suppose that $E\left(\left[f_{1}\right]\right)=$ $E\left(\left[f_{2}\right]\right)$. As $c^{*}$ is injective, it follows that $\left[f_{1}\right]$ and $\left[f_{2}\right]$ have the same normal invariant and so there is an element $x \in L_{n+l+1}(\pi)$ such that $\left[f_{1}\right]=\left[f_{2}\right]+x$ where + denotes the action of $L_{n+l+1}(\pi)$ on $\mathcal{S}_{\partial}\left(M \times D^{l}\right)$. Hence $E\left(\left[f_{1}\right]\right)=E\left(\left[f_{2}\right]\right)+x$ and hence $x$ acts trivially on $E\left(\left[f_{1}\right]\right)=E\left(\left[f_{2}\right]\right)$.

Now we use the fact that the topological surgery exact sequence is a long exact sequence of abelian groups by [31, C.5; 26, Theorem 18.5]. It follows that an element $y \in$ $L_{n+l+1}(\pi)$ acts trivially on an element of $\mathcal{S}(T)$ if and only if $y \in \operatorname{Ker}\left(\omega_{2}\right)=\operatorname{Im}\left(\sigma_{2}\right)$ and similarly for $\mathcal{S}_{\partial}\left(M \times D^{l}\right)$. We deduce that $x \in \operatorname{Im}\left(\sigma_{2}\right)$ and that it remains to show that $x \in \operatorname{Im}\left(\sigma_{1}\right)$, ie that $\operatorname{Im}\left(\sigma_{2}\right)=\operatorname{Im}\left(\sigma_{1}\right)$.

By the commutativity of the above diagram $\operatorname{Im}\left(\sigma_{1}\right) \subset \operatorname{Im}\left(\sigma_{2}\right)$. On the other hand, we see that

$$
\left[\Sigma^{1} T_{+}, G / \mathrm{TOP}\right] \cong \operatorname{Im}\left(\left(\Sigma^{1} c\right)^{*}\right) \oplus \operatorname{Im}\left(\left(\Sigma^{1} p\right)^{*}\right) .
$$

Now the geometric description of $\left(\Sigma^{1} p\right)^{*}$ is to multiply a degree one normal map to $M \times[0,1]$ by the identity map of $S^{l}$ and moreover $S^{l}$ has trivial symmetric signature. Using a simple modification of [25, Proposition 8.1] to the relative case we conclude that $\sigma_{2}$ vanishes on $\operatorname{Im}\left(\left(\Sigma^{1} p\right)^{*}\right)$. It follows that $\operatorname{Im}\left(\sigma_{2}\right)=\operatorname{Im}\left(\sigma_{1}\right)$ and hence $E$ is injective.

Equation (4-7) and Lemma 4.5 yield:

Corollary 4.7 There is an equality $\left[\left(h_{0}, \omega_{0}, H_{0}\right)^{\prime}\right]=\left[\left(h_{1}, \omega_{0},\left.H_{0} \cup G\right|_{X}\right)^{\prime}\right]$.

Lemma 4.4 and Corollary 4.7 show that the map $\mathrm{CW}^{k / 2}$ is well defined.

Remark 4.8 The discussion above includes, in particular, a proof of [11, Lemma 1.1]: for a fixed choice of $\omega$, a choice of homotopy $H$ does not effect the equivalence class of $(h, \omega, H)^{\prime}: N^{\prime} \rightarrow M \times D^{2 k}$ in $\mathcal{S}_{\partial}\left(M \times D^{2 k}\right)$. The proof of this in the appendix 
of [11] appears to us to be incorrect: the claim in the first paragraph of Hutt's Appendix that there exists a homotopy $K$ between the homotopy $-H_{2}+H_{1}$ and an isotopy $\mathcal{H}$ between $\psi_{2}$ and $\psi_{1}$ is not justified.

\subsection{Extension by homeomorphism}

Recall that $M$ is a closed topological manifold, that $\mathbb{F}=\mathbb{C}$ or $\mathbb{H}$ has real dimension $k=2$ or 4 and consider the obvious decomposition

$$
M \times \mathbb{F} P^{2}=\left(M \times D^{2 k}\right) \cup\left(M \times \mathbb{F} P^{2 \bullet}\right)
$$

where $\mathbb{F} P^{2 \bullet}:=\mathbb{F} P^{2}-D^{2 k}$. Associated to this decomposition extension by homeomorphism gives the map of structure sets

$$
E: \mathcal{S}_{\partial}\left(M \times D^{2 k}\right) \rightarrow \mathcal{S}\left(M \times \mathbb{F} P^{2}\right) .
$$

For the structures $h^{\prime}: N^{\prime} \rightarrow M \times D^{2 k}$ defined in (4-5) above, we can realize the map $E$ as follows. Observe that $\mathbb{F} P^{2 \bullet}$ is homeomorphic to the mapping cylinder (disk bundle) of the Hopf map $\gamma: S^{2 k-1} \rightarrow S^{k}$ and recall that the restriction of the map $h^{\prime}$ to $\partial N^{\prime}=\partial W^{\prime}$ is given by the $S^{k-1}$-bundle map $\partial \psi^{\prime}: \partial W^{\prime} \rightarrow M \times S^{2 k-1}$ over $\partial \psi: \partial W \rightarrow M \times S^{k}$. Thus we can simply extend $\partial \psi^{\prime}$ to the associated $D^{k}$-bundle map. This amounts to extending to the associated mapping cylinders.

To be explicit, recall that $q_{W}: \partial W^{\prime} \rightarrow \partial W$ is the projection in the source and define

$$
\widehat{N}:=N^{\prime} \cup_{\partial W^{\prime}} \operatorname{cyl}\left(q_{W}\right)=\operatorname{cyl}\left(p^{\prime}\right) \cup U_{\partial}^{\prime} \cup_{\partial W^{\prime}} \operatorname{cyl}\left(q_{W}\right) .
$$

If follows that a representative of $E\left[h^{\prime}\right]$ given by

$$
\widehat{h}:=h^{\prime} \cup \operatorname{cyl}\left(\partial \psi^{\prime}, \partial \psi\right): \widehat{N} \rightarrow M \times \mathbb{F} P^{2}
$$

where cyl $\left(\partial \psi^{\prime}, \partial \psi\right)$ is the mapping cylinder $\left(D^{k}\right.$-bundle $)$ map associated to the square $\partial \psi \circ q=\gamma \circ \partial \psi^{\prime}$.

As with the map $h^{\prime}$ we note that the map $\widehat{h}: \widehat{N} \rightarrow M \times \mathbb{F} P^{2}$ is not a map over $h: N \rightarrow M$ since $\partial \psi$ is not a map over $h$. On the other hand, we can replace the structure $\hat{h}$ with a structure that is over $h$. In fact one has the following h-decorated version of a key lemma of Hutt.

Lemma 4.9 (Compare [11, Lemma 1.4].) There is a closed manifold $\bar{N}$ with a map $\bar{p}: \bar{N} \rightarrow N$ and a homotopy equivalence of closed manifolds $\bar{h}: \bar{N} \rightarrow M \times \mathbb{F} P^{2}$ covering the map $h$ which represents the same element as $\hat{h}$ in $\mathcal{S}\left(M \times \mathbb{F} P^{2}\right)$. Indeed 
there is a homotopy equivalence $\varphi: \bar{N} \rightarrow N \times \mathbb{F} P^{2}$ over $N$ such that the following diagram commutes.

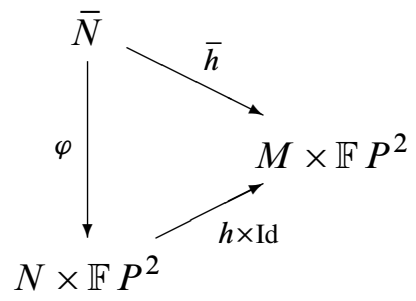

Proof Recall the projection map $q \bar{W}: \partial \bar{W}^{\prime} \rightarrow \partial \bar{W}$ and also recall that the mapping cylinder cyl $\left(p^{\prime}\right)$ has boundary $\partial \bar{W}^{\prime}$. The manifold $\bar{N}$ is defined as

$$
\bar{N}:=\operatorname{cyl}\left(p^{\prime}\right) \cup \operatorname{cyl}(q \bar{W})
$$

and we have the obvious projection map $\bar{p}: \bar{N} \rightarrow N$. Recall the homotopy equivalence $\omega: \bar{W} \rightarrow N \times D^{k+1}$ over the identity on $N$ from Lemma 4.1. Define $\varphi: \bar{N} \simeq N \times \mathbb{F} P^{2}$ by

$$
\varphi=\operatorname{cyl}\left(\partial \omega^{\prime}, \mathrm{id}\right) \cup \operatorname{cyl}\left(\partial \omega^{\prime}, \partial \omega\right),
$$

and $\bar{h}=(h \times \mathrm{Id}) \circ \varphi: \bar{N} \simeq M \times \mathbb{F} P^{2}$.

We need to show that $\bar{h}$ is equivalent to $\hat{h}$ and we achieve this with a sort of Alexander trick. We first find an $h$-cobordism $Z$ between the manifolds $\hat{N}$ and $\bar{N}$. To this end think of $\bar{N}$ as

$$
\bar{N}=\operatorname{cyl}\left(p^{\prime}\right) \cup(\partial \bar{W} \times[0,1]) \cup \operatorname{cyl}(q \bar{W}) .
$$

Now the manifold $Z_{3}:=\operatorname{cyl}\left(q_{U_{\partial}}\right)$ yields an $h$-cobordism between $\operatorname{cyl}\left(q_{W}\right)$ and $\operatorname{cyl}(q \bar{W})$. The product $U_{\partial}^{\prime} \times[0,1]$ can be viewed as an $h$-cobordism $Z_{2}$ between $U_{\partial}^{\prime}$ and $\partial \bar{W}^{\prime} \times[0,1]$. Let $Z_{1}$ be the trivial product $h$-cobordism over cyl $\left(p^{\prime}\right)$. Gluing all these together gives the desired global $h$-cobordism.

It remains to produce a homotopy equivalence from $Z$ to $M \times \mathbb{F} P^{2} \times[0,1]$ which restricts to $\hat{h}$ and $\bar{h}$ on the two ends. On $Z_{1}$ we just take the product of $\operatorname{cyl}\left(\partial \bar{\psi}^{\prime}, \partial \bar{\psi}\right)$ with the identity. On $Z_{2}$ we take the product of $H_{\partial}^{\prime}$ with the identity, but this is modified according to the way we think of $Z_{2}$. On $Z_{3}$ we can take the map cyl $\left(H_{\partial}^{\prime}, H_{\partial}\right)$. All these maps agree on the boundary and provide the required homotopy equivalence.

Remark 4.10 Notice that the only role played by $h$ in the construction of the map $\varphi$ is to define $\chi \in[N, \mathrm{G} / \mathrm{TOP}]$. Specifically $\varphi$ is determined by $\partial \omega$ and $\partial \omega$ is determined by $\chi \in[N ; \mathrm{G} / \mathrm{TOP}]$. Moreover the class $\chi$ is all one needs to build the manifolds $N^{\prime}$, $\hat{N}$ and $\bar{N}$. This observation will be useful in the last Section 8 where coboundaries for $\hat{N}$ and $\bar{N}$ will be constructed. 


\section{The algebraic theory of surgery}

We give a brief review of the algebraic theory of surgery. In particular we review how algebraic surgery equips $\mathcal{S}(M)$ with an abelian group structure by identifying it with the algebraic structure set $\mathbb{S}_{n+1}(M)$. In more detail, the aim of this section is to define, for a closed $n$-dimensional topological manifold, the abelian algebraic structure group $\mathbb{S}_{n+1}(M)$ and the map $s: \mathcal{S}(M) \rightarrow \mathbb{S}_{n+1}(M)$ which turns out to be a bijection if $n \geq 5$. Hence one can equip $\mathcal{S}(M)$ with an abelian group structure via this bijection. We will also discuss a generalization when $M$ has a boundary. Furthermore we will discuss the functoriality of $\mathbb{S}_{n+1}(M)$ in $M$ and we will present a condition which implies that an element in $\mathbb{S}_{n+1}(M)$ is zero. All these results will be used in subsequent sections. The principal references are Ranicki's papers [26; 24; 25; 28].

The abelian group $\mathbb{S}_{n+1}(M)$ is defined as a quadratic $L$-group of a certain algebraic bordism category.

An algebraic bordism category $\Lambda=(\mathbb{A}, \mathbb{B}, \mathbb{C})$ consists of an additive category with chain duality $(\mathbb{A},(T, e))$ and two full subcategories $\mathbb{C}, \mathbb{B} \subseteq \mathbb{B}(\mathbb{A})$ of the category of bounded chain complexes in $\mathbb{A}$ satisfying certain mild assumptions.

The chain duality $(T, e)$ consists of a contravariant functor $T: \mathbb{B}(\mathbb{A}) \rightarrow \mathbb{B}(\mathbb{A})$ and a natural transformation $e: T^{2} \rightarrow$ id satisfying certain conditions. It allows one to define the tensor product $C \otimes_{\mathbb{A}} D$ of chain complexes $C, D \in \mathbb{B}(\mathbb{A})$ such that the tensor product $C \otimes_{\mathbb{A}} C$ becomes a chain complex of $\mathbb{Z}\left[\mathbb{Z}_{2}\right]$-modules. One defines an $n-$ dimensional symmetric structure on $C \in \mathbb{B}(\mathbb{A})$ to be an $n$-cycle $\phi$ in the chain complex $W^{\%}(C)=\operatorname{Hom}_{\mathbb{Z}\left[\mathbb{Z}_{2}\right]}\left(W,\left(C \otimes_{\mathbb{A}} C\right)\right)$, where $W$ is the standard $\mathbb{Z}\left[\mathbb{Z}_{2}\right]$-resolution of $\mathbb{Z}$. An $n$-dimensional quadratic structure on $C \in \mathbb{B}(\mathbb{A})$ is defined as an $n$-cycle $\psi$ in the chain complex $W_{\%}(C)=W \otimes_{\mathbb{Z}\left[\mathbb{Z}_{2}\right]}\left(C \otimes_{\mathbb{A}} C\right)$. An $n$-dimensional symmetric structure consists of a collection of chains $\phi=\left\{\phi_{s} \in\left(C \otimes_{\mathbb{A}} C\right)_{n+s} \mid s \geq 0\right\}$ satisfying certain compatibility connections. An $n$-dimensional quadratic structure consists of a collection of chains $\psi=\left\{\psi_{s} \in\left(C \otimes_{\mathbb{A}} C\right)_{n-s} \mid s \geq 0\right\}$ satisfying certain compatibility connections. There is also a symmetrization map $(1+T)$ : $W_{\%}(C) \rightarrow W^{\%}(C)$.

The pair $(C, \phi)$ is called a symmetric algebraic complex, it is $\mathbb{C}$-Poincare if the mapping cone of $\phi_{0}, \mathcal{C}\left(\phi_{0}: \Sigma^{n} T C \rightarrow C\right)$, lies in $\mathbb{C}$. The pair $(C, \psi)$ is called a quadratic algebraic complex, it is $\mathbb{C}$-Poincaré if $(C,(1+T) \psi)$ is $\mathbb{C}$-Poincaré. In the above formula recall that

$$
\left(C \otimes_{\mathbb{A}} C\right)_{n}:=\operatorname{Hom}_{\mathbb{A}}(T C, C)_{n}=\operatorname{Hom}_{\mathbb{A}}\left(\Sigma^{n} T C, C\right)_{0} .
$$

All these notions are defined in [26, Chapters 1,3]. 
$L$-Groups There is a well-defined notion of a cobordism of $n$-dimensional quadratic algebraic complexes. The quadratic $L$-groups $L_{n}(\Lambda)$ are the cobordism group of $n$-dimensional algebraic complexes in $\Lambda$, that means elements are represented by those complexes which are in $\mathbb{B} \subset \mathbb{B}(\mathbb{A})$ which are $\mathbb{C}$-Poincaré. If $\mathbb{B}$ and $\mathbb{C}$ are not explicitly stated, we use the convention that $\mathbb{B}=\mathbb{B}(\mathbb{A})$ and $\mathbb{C}=0$. See $[26$, Definitions $1.8,3.4]$.

Example $\mathbb{A}\left[\pi_{1}(M)\right]$ For any ring with involution $R$, for example for $\mathbb{Z}\left[\pi_{1}(M)\right]$, the category of finitely generated free based $R$-modules $\mathbb{A}[R]$ has a chain duality given by $T(M)=\operatorname{Hom}_{R}(M, R)$. Examples 5.3, 5.4 below explain how to obtain symmetric and quadratic algebraic complexes over the category $\mathbb{Z}\left[\pi_{1}(M)\right]$. The quadratic $L-$ groups $L_{n}\left(\mathbb{Z}\left[\pi_{1}(M)\right]\right)$ defined as cobordism groups of quadratic algebraic Poincaré complexes agree with the usual Wall surgery $L$-groups defined using quadratic forms or formations [25].

Example $\mathbb{A}_{*}(K)$ Let $K$ be a simplicial complex, or more generally a $\Delta$-set, and let $\mathbb{A}$ be an additive category with chain duality. The category $\mathbb{A}_{*}(K)$ has as its objects the so-called $K$-based objects of $\mathbb{A}$, that means objects of $\mathbb{A}$ which come as direct sums

$$
M=\sum_{\sigma \in K} M(\sigma)
$$

Morphisms are given by

$\operatorname{Mor}_{\mathbb{A}_{*}(K)}(M, N)=\left\{f=\sum_{\sigma, \tau \in K} f(\tau, \sigma): M(\sigma) \rightarrow N(\tau) \mid f(\tau, \sigma)=0\right.$ unless $\left.\sigma \leq \tau\right\}$.

The definition of the duality is stated in [26, Proposition 5.1], on the objects $M \in \mathbb{A}$ it is given by

$$
(T M)_{r}(\sigma)=T(M(\sigma))_{r+|\sigma|} \quad \text { if } \quad \sigma \leq \tau,|\sigma|=|\tau|-1 .
$$

This formula will not be used in the present paper. What is more important for us is the observation that an $n$-dimensional quadratic algebraic complex $(C, \psi)$ in $\mathbb{A}_{*}(K)$ includes in particular for each $\sigma \in K$ a chain complex $C(\sigma)$ and a duality map $\psi_{0}(\sigma): \Sigma^{n} T C(\sigma) \rightarrow C(\sigma)$ (recall (5-1)). But it contains more information. There are relations between these data for various simplices and of course the components $\psi_{s}$ for $s>0$. See [26, Definition 4.1, Proposition 5.1]. Also Examples 5.3, 5.5 below explain how such complexes come from geometry. 
Functoriality Let $\pi: K \rightarrow L$ be a $\Delta$-set map. Then we have an additive functor

$$
\pi_{*}: \mathbb{A}_{*}(K) \rightarrow \mathbb{A}_{*}(L) \quad\left(\pi_{*} M\right)(\tau)=\sum_{\sigma \in K, \pi(\sigma)=\tau} M(\sigma) \quad \text { for } \tau \in L
$$

which induces a functor on the chain complexes which also "commutes" with the chain duality in a suitable sense so that one obtains a map of the $L$-groups

$$
\pi_{*}: L_{n+1}\left(\mathbb{A}_{*}(K)\right) \rightarrow L_{n+1}\left(\mathbb{A}_{*}(L)\right) .
$$

See [26, Proposition 5.6, Example 5.8].

Assembly Slightly different functoriality is provided by the assembly functor denoted $A: \mathbb{Z}_{*}(K) \rightarrow \mathbb{Z}\left[\pi_{1}(K)\right]$ and defined by

$$
M \mapsto \sum_{\tilde{\sigma} \in \widetilde{K}}=M(p(\widetilde{\sigma}))
$$

which also induces a map of the $L$-groups

$$
\pi_{*}: L_{n}\left(\mathbb{Z}_{*}(K)\right) \cong H_{n}\left(K, \mathbf{L}_{\bullet}\right) \rightarrow L_{n}\left(\mathbb{Z}\left[\pi_{1}(K)\right]\right) .
$$

Here the isomorphism in the source is a calculation, the symbol $\mathbf{L}_{\bullet}$ denotes the quadratic $\langle 0\rangle$-connective $L$-theory spectrum. See [26, Chapter 9; 26, Chapter 13] for the spectrum $\mathbf{L}$.

Algebraic bordism categories So far we have only presented examples of additive categories with chain duality. In order to obtain an algebraic bordism category we need to specify interesting subcategories of $\mathbb{B}(\mathbb{A})$. We will only be interested in the case $\mathbb{A}=\mathbb{A}_{*}(K)$ for which we will use three such subcategories, denoted $\mathbb{D} \subset \mathbb{C} \subset \mathbb{B}$. Here are the definitions:

$$
\begin{aligned}
& \mathbb{B}:=\mathbb{B}\left(\mathbb{A}_{*}(K)\right), \\
& \mathbb{C}:=\{C \in \mathbb{B} \mid A(C) \simeq *\}, \\
& \mathbb{D}:=\{C \in \mathbb{B} \mid C(\sigma) \simeq * \forall \sigma \in K\} .
\end{aligned}
$$

This gives us three possibilities to construct interesting algebraic bordism categories. Using a suitable notion of a functor between algebraic bordism categories we obtain a sequence

$$
\Lambda^{\prime \prime}=(\mathbb{A}, \mathbb{C}, \mathbb{D}) \longrightarrow \Lambda^{\prime}=(\mathbb{A}, \mathbb{B}, \mathbb{D}) \longrightarrow \Lambda=(\mathbb{A}, \mathbb{B}, \mathbb{C})
$$

which induces a long exact sequence of groups

$$
\cdots \rightarrow L_{n+1}(\Lambda) \rightarrow L_{n}\left(\Lambda^{\prime \prime}\right) \rightarrow L_{n}\left(\Lambda^{\prime}\right) \rightarrow L_{n}(\Lambda) \rightarrow L_{n-1}\left(\Lambda^{\prime \prime}\right) \rightarrow \cdots .
$$

This material is discussed in [26, Chapter 3]. 
Connective versions In order to obtain groups which relate well with geometry we need to use a connective version of the above theory. Let $q \in \mathbb{Z}$ and let $\Lambda=(\mathbb{A}, \mathbb{B}, \mathbb{C})$ be an algebraic bordism category. Define the subcategory $\mathbb{B}\langle q\rangle \subset \mathbb{B}$ to be the subcategory of chain complexes in $\mathbb{B}$ which are homotopy equivalent to $q$-connected chain complexes. Further define $\mathbb{C}\langle q\rangle=\mathbb{B}\langle q\rangle \cap \mathbb{C}$. Then $\Lambda\langle q\rangle=(\mathbb{A}, \mathbb{B}\langle q\rangle, \mathbb{C}\langle q\rangle)$ is a new algebraic bordism category. More details are given in [26, Chapter 15].

Definition 5.1 [26, Chapter 17] Let $K$ be a $\Delta$-set, $n \in \mathbb{N}$ and let $\Lambda^{\prime \prime}$ be the algebraic bordism category given by (5-4). Define

$$
\mathbb{S}_{n+1}(K):=L_{n}\left(\Lambda_{*}^{\prime \prime}\langle 1\rangle\right) \text {. }
$$

Algebraic surgery exact sequence Putting together the previous statements and definitions the long exact sequence (5-5) becomes the algebraic surgery exact sequence

$$
\cdots \rightarrow L_{n+1}\left(\mathbb{Z}\left[\pi_{1}(K)\right]\right) \rightarrow \mathbb{S}_{n+1}(K) \rightarrow H_{n}(K, \mathbf{L} \bullet\langle 1\rangle) \rightarrow L_{n}\left(\mathbb{Z}\left[\pi_{1}(K)\right]\right) \rightarrow \cdots
$$

discussed in detail in [26, Chapters 14,15$]$. We will mostly work directly with the group $\mathbb{S}_{n+1}(K)$, but of course many of the properties of this group follow from the existence of the sequence (5-6). For example recall that the assignment $K \mapsto \mathbb{S}_{n+1}(K)$ becomes a covariant functor from $\Delta$-sets to abelian groups via the functoriality described in (5-2). So for $\pi: K \rightarrow L$ a $\Delta$-set map we obtain the map

$$
\pi_{*}: \mathbb{S}_{n+1}(K) \rightarrow \mathbb{S}_{n+1}(L) .
$$

In fact we obtain a map of exact sequences for $K$ and $L$ and it follows that the functor $\mathbb{S}_{n+1}(K)$ is a homotopy functor. In particular this means that if $\pi$ is a homotopy equivalence of $\Delta$-sets, then $\pi_{*}$ is an isomorphism of abelian groups, since the other two terms clearly are homotopy functors.

Next we review how the above theory relates to topology. We begin with some remarks about topological manifolds in the above setting. When $M$ is a closed $n$-dimensional topological manifold we can apply Definition 5.1 only if $M$ is triangulated. In that case it is possible to define a map $s: \mathcal{S}(M) \rightarrow \mathbb{S}_{n+1}(M)$, whose construction we recall below and which can be shown to be a bijection [26, Chapter 18]. If $M$ is not triangulated we can choose a homotopy equivalence $r: M \rightarrow K$ to a finite $\Delta$-set. Such an $r$ will determine a map $s(r): \mathcal{S}(M) \rightarrow \mathbb{S}_{n+1}(K)$ which can be shown to be a bijection in the same way. In both cases the bijections $s$ and $s(r)$ can be used to give $\mathcal{S}(M)$ the structure of an abelian group. It also turns out that this group structure is independent of both the choice of the triangulation and of the homotopy equivalence $r$. Therefore, following Ranicki, we will abuse notation and write $\mathbb{S}_{n+1}(M)$ for $\mathbb{S}_{n+1}(K)$ for any choice of a $\Delta$-set $K$ homotopy equivalent to $M$ and $s: \mathcal{S}(M) \rightarrow \mathbb{S}_{n+1}(M)$ 
for $s(r)$ given by any choice of a homotopy equivalence $r: M \rightarrow K$. For the record we make:

Definition 5.2 Let $M$ be an $n$-dimensional topological manifold and let $r: M \rightarrow K$ be a homotopy equivalence to a finite $\Delta$-set. We write

$$
\mathbb{S}_{n+1}(M)=\mathbb{S}_{n+1}(K) \text {. }
$$

Now we explain the map $s: \mathcal{S}(M) \rightarrow \mathbb{S}_{n+1}(M)$. First we need some preparation. In the following examples we explain the topological situations which give rise to algebraic complexes, both symmetric and quadratic.

Example 5.3 Let $K$ be a finite $\Delta$-set with barycentric subdivision $K^{\prime}$. Consider the simplicial chain complex $C:=\Delta_{*}\left(K^{\prime}\right)$ as an object in $\mathbb{B}(\mathbb{Z})$. Any $n$-cycle $[K]$ in $C_{n}$ defines via the symmetric construction a symmetric structure $\phi$ on $C$ over $\mathbb{Z}$, whose component $\phi_{0}: \Sigma^{n} T C=C^{n-*} \rightarrow C_{*}$ corresponds to the cap product with $[K]$. If $K$ is a closed oriented $n$-dimensional topological manifold and $[K]$ is its fundamental class, then the resulting symmetric Poincaré complex is denoted $\sigma^{*}(K)$ and is called the symmetric signature of $K$. See [25]

The chain complex $C$ can also be thought of as an object in $\mathbb{B}\left(\mathbb{Z}_{*}(K)\right)$ with $C(\sigma)=$ $\Delta_{*}(D(\sigma), \partial D(\sigma))$, the simplicial chain complex of the dual cell relative to its boundary. Then we have $\Sigma^{n} T C(\sigma) \cong \Delta^{n-|\sigma|-*}(D(\sigma))$. Again by [26] for each $n$-cycle $[K] \in C_{n}$ there is a refined symmetric construction. Thus we obtain an algebraic symmetric structure $\phi$ over $\mathbb{Z}_{*}(K)$, which in particular contains for each $\sigma$ duality maps $\phi_{0}(\sigma): \Sigma^{n} T C(\sigma) \rightarrow C(\sigma)$ which are cap products with certain $(n-|\sigma|)$-dimensional classes $[K](\sigma)$. See [26, Example 5.5].

Now let $M$ be a topological manifold with a reference homotopy equivalence $r: M \rightarrow K$ to a $\Delta$-set, which is transverse to the dual cells of $K^{\prime}$. Consider the dissection

$$
M=\bigcup_{\sigma \in K}\left(M(\sigma)=r^{-1}(D(\sigma)) .\right)
$$

The chain complex

$$
C=\sum_{\sigma \in K}(C(\sigma)=C(M(\sigma), \partial M(\sigma)))
$$

where $C(M(\sigma), \partial M(\sigma))$ is the singular chain complex of the pair $(M(\sigma), \partial M(\sigma))$, yields an object in $\mathbb{B}\left(\mathbb{Z}_{*}(K)\right)$. As an object in $\mathbb{B}(\mathbb{Z})$ it is weakly homotopy equivalent to $C_{*}(M)$, the singular chain complex of $M$. When considered as an object in $\mathbb{B}\left(\mathbb{Z}_{*}(K)\right)$, then, similarly to above, there is for each $n$-cycle $[M] \in C_{n}$ a refined symmetric 
construction, so that we obtain an algebraic symmetric structure $\phi$ over $\mathbb{Z}_{*}(K)$, which in particular contains for each $\sigma$ duality maps $\phi_{0}(\sigma): \Sigma^{n} T C(\sigma) \rightarrow C(\sigma)$ which are cap products with certain $(n-|\sigma|)$-dimensional classes $[M](\sigma)$. For more details see [26, Example 6.2].

Example 5.4 Let $(f, b): N \rightarrow M$ be a degree one normal map of $n$-dimensional closed manifolds. Denote by $K(f)$ the algebraic mapping cone of the Umkehr map of chain complexes

$$
f^{!}: C_{*} \widetilde{M} \simeq C^{n-*} \widetilde{M} \stackrel{f^{n-*}}{\longrightarrow} C^{n-*} \tilde{N} \simeq C_{*} \tilde{N} .
$$

Then $C_{*} \widetilde{M}$ comes with a structure of an $n$-dimensional symmetric algebraic Poincaré complex over $\mathbb{Z}\left[\pi_{1}(M)\right]$. This projects to a structure of an $n$-dimensional symmetric algebraic Poincaré complex on $K(f)$. In [25] this is refined to an $n$-dimensional quadratic algebraic Poincaré complex on $(K(f), \psi(f))$.

Example 5.5 Let $(f, b): N \rightarrow M$ be a degree one normal map of closed $n$-dimensional manifolds and let $r: M \rightarrow K$ be a map to a $\Delta$-set $K$ such that both $r f$ and $r$ are transverse to the dual cells of $K$. There are $K$-dissections $N \cong \cup N(\sigma)$ and $M \cong \cup M(\sigma)$, so that $C_{*} N$ and $C_{*} M$ can be regarded as objects in $\mathbb{B}\left(\mathbb{Z}_{*}(K)\right)$. There are preferred structures of $n$-dimensional symmetric algebraic complexes on $C_{*} N$ and $C_{*} M$, as objects of $\mathbb{B}\left(\mathbb{Z}_{*}(K)\right)$ coming from the fundamental classes. By analogy with Example 5.4, there is an algebraic Umkehr map

$$
f^{!}: C_{*} M \longrightarrow C_{*} N
$$

in $\mathbb{B}\left(\mathbb{Z}_{*}(K)\right)$ with mapping cone $K(f)$, say. The resulting structure of and $n-$ dimensional symmetric algebraic complex on $K(f)$, as an object of $\mathbb{B}\left(\mathbb{Z}_{*}(K)\right)$, has a preferred refinement to a quadratic structure $\psi(f)$. The chain complex $K(f)(\sigma)$ for a $\sigma \in K$ can be identified with the mapping cone of an algebraic Umkehr map

$$
C_{*}(M(\sigma), \partial M(\sigma)) \longrightarrow C_{*}(N(\sigma), \partial N(\sigma)) .
$$

See [26, Examples 9.13, 9.14] for details. Under assembly, this construction coincides with that in Example 5.4.

Example 5.6 Note that $(K(f), \psi(f))$ is $\mathbb{D}$-Poincaré. When in addition $f$ is a homotopy equivalence, then $K(f)$ is contractible after assembly. Furthermore the required connectivity assumptions are fulfilled so that the pair $(K(f), \psi(f))$ represents an element in $\mathbb{S}_{n+1}(M)$. 
Definition 5.7 [26, Proposition 18.3] The map

$$
s: \mathcal{S}(M) \rightarrow \mathbb{S}_{n+1}(M), \quad[f: N \rightarrow M] \mapsto[K(f), \psi(f)]
$$

is defined by the construction described in Examples 5.5, 5.6.

Example 5.8 [26, Proposition 18.3] In case we deal with a manifold with boundary $(Y, \partial Y)$, the constructions in Examples 5.5, 5.6 yield a map from $\mathcal{S}_{\partial}(Y)$ to $\mathbb{S}_{n+1}(Y)$. When $Y=M \times D^{k}$, then thanks to the homotopy invariance of $\mathbb{S}_{*}(-)$ we obtain a map

$$
s: \mathcal{S}_{\partial}\left(M \times D^{k}\right) \rightarrow \mathcal{S}_{n+1+k}(M)
$$

Theorem 5.9 [26, Theorem 18.5] For a closed manifold $M$ with dimension $n \geq 5$ we have

$$
s: \mathcal{S}(M) \stackrel{\equiv}{\rightrightarrows} \mathbb{S}_{n+1}(M), \quad s: \mathcal{S}_{\partial}\left(M \times D^{k}\right) \stackrel{\cong}{\rightarrow} \mathcal{S}_{n+1+k}(M),
$$

where $\equiv$ means a bijection and $\cong$ means an isomorphism of abelian groups.

Remark 5.10 Suppose that $h: M \rightarrow M^{\prime}$ is a homotopy equivalence of $n$-dimensional closed manifolds. Then the assignment $[f] \mapsto[h \circ f]$ provides us with a map of sets $\mathcal{S}(M) \rightarrow \mathcal{S}\left(M^{\prime}\right)$. On the other hand $h$ induces a homomorphism $h_{*}: \mathbb{S}_{n+1}(M) \rightarrow$ $\mathbb{S}_{n+1}\left(M^{\prime}\right)$. We note that in general $s([h \circ f]) \neq h_{*}([f])$, but there is the composition formula $s([h \circ f])=h_{*} s([f])+[h]$; see [27]. This will indeed be used later in the proof of Proposition 6.1.

Example 5.11 Let $h: P \rightarrow N$ be a homotopy equivalence of closed $n$-dimensional manifolds representing an element in $\mathcal{S}(N)$. Given a homotopy equivalence $r: N \rightarrow K$ to a finite $\Delta$-set $K$ we have described $s([h]) \in \mathbb{S}_{n+1}(N)$ in Examples 5.5, 5.6. Let $s: M \rightarrow L$ be a homotopy equivalence from another closed $n$-dimensional manifold to a finite $\Delta$-set and let $f: N \rightarrow M$ be a map covering via the reference maps a $\Delta$-set map $\pi: K \rightarrow L$. Consider $\pi_{*}(s([h])) \in \mathbb{S}_{n+1}(M)$. From the description of the functoriality in 5.11 we see that for each $\tau \in L, \pi_{*}(s([h]))(\tau)$ has the underlying chain complex the algebraic mapping cone of the map

$$
\left(\bigcup_{\pi(\sigma)=\tau} P(\sigma), \partial\right) \longrightarrow\left(\bigcup_{\pi(\sigma)=\tau} N(\sigma), \partial\right) .
$$

See [26, Example 5.8]. 
Example 5.12 We will need a special case of the above example when $\pi$ is the projection map $\pi_{1}: K \otimes L \rightarrow K$. Here $K \otimes L$ is the geometric product of $\Delta$-sets; see [30; 26, Chapter 11]. A $p$-simplex of $K \otimes L$ is a triple

$$
(\sigma, \tau, \lambda) \quad \text { where } \quad \sigma \in K^{(m)}, \tau \in L^{(n)}, \lambda \in\left(\Delta^{m} \otimes \Delta^{n}\right)^{(p)}
$$

with $\Delta^{m} \otimes \Delta^{n}$ the product of ordered simplicial complexes. There is a homeomorphism $|K \otimes L|=|K| \times|L|$ and there is a projection map $\pi_{1}: K \otimes L \rightarrow L$ which is a $\Delta$-set map (the explicit formula is easy but a little complicated and we do not need it). We have

$$
\bigcup_{\tau, \lambda} D(\sigma, \tau, \lambda)=D(\sigma) \times L
$$

Let $M$ and $N$ be two closed topological manifolds with reference homotopy equivalences to $\Delta$-sets $r: M \rightarrow K$ and $r^{\prime}: N \rightarrow L$ transverse to the dual cells. Then the product map $r \times r^{\prime}: M \times N \rightarrow|K| \times|L|$ is transverse to the dual cells of the geometric product of the $\Delta$-sets $K \otimes L$. Let $h: P \rightarrow M \times N$ be a simple homotopy equivalence representing an element in $\mathcal{S}(M \times N)$ which is transverse to the dissection of $M \times N$ induced by $r \times r^{\prime}$. We have $s([h]) \in \mathbb{S}_{m+n+1}(M \times N)$ and this element is represented by an algebraic Poincaré complex over $\mathbb{Z}_{*}(K \otimes L)$ whose value at $(\sigma, \tau, \lambda) \in K \otimes L$ has its underlying chain complex the mapping cone of the Umkehr map of the degree one normal map

$$
h(\sigma, \tau, \lambda): P(\sigma, \tau, \lambda)=h^{-1}(M \times N)(\sigma, \tau, \lambda) \rightarrow(M \times N)(\sigma, \tau, \lambda) .
$$

Consider the projection map $p: M \times N \rightarrow M$. We have $p_{*}(s([h])) \in \mathbb{S}_{m+n+1}(M)$. It follows from the above discussion that this element is represented by an algebraic Poincaré complex in $\mathbb{A}_{*}(K)$ whose value at $\sigma \in K$ has its underlying chain complex the mapping cone of the Umkehr map of the degree one normal map

$$
h(\sigma): P(\sigma)=h^{-1}(M(\sigma) \times N) \rightarrow M(\sigma) \times N .
$$

It may happen that such $h$ represents a nontrivial element in $\mathcal{S}(M \times N)$ and hence $s([h])$ is a nonzero element in $\mathbb{S}_{m+n+1}(M \times N)$ and at the same time for each $\sigma \in K$ the map (5-10) is a simple homotopy equivalence. Then the underlying chain complex for each $\sigma$ is contractible and hence the projection $p_{*}(s([h]))=0 \in \mathbb{S}_{m+n+1}(M)$ by the Proposition 5.13 below. Such a situation will indeed occur in the next section.

Proposition 5.13 Let $(C, \psi)$ represent an element in $\mathbb{S}_{n+1}(M)$. Suppose in addition that $C(\sigma) \simeq *$ for each $\sigma \in K$. Then

$$
[(C, \psi)]=0 \in \mathbb{S}_{n+1}(M) .
$$


Proof The homotopy equivalences for each $\sigma$ assemble to a null-bordism of chain complexes in the algebraic bordism category $\Lambda^{\prime \prime}\langle 1\rangle$ with $\Lambda^{\prime \prime}=\left(\mathbb{Z}_{*}(K), \mathbb{C}, \mathbb{D}\right)$ as in (5-3) which defines $\mathbb{S}_{n+1}(M)$.

One of the important and useful features of the algebraic theory of surgery is a particularly easy description of periodicity. Indeed, in case one works with the 0 connective version of the algebraic structure set, denoted by $\overline{\mathbb{S}}_{n+1}(M)$, one obtains the 4-periodicity given by the so-called skew-double-suspension

$$
\bar{S}^{2}: \overline{\mathbb{S}}_{n+1}(M) \rightarrow \overline{\mathbb{S}}_{n+5}(M) \text {. }
$$

If one works with the 1 -connective version and for a positive integer $k$ sets $S^{2 k}:=$ $\left(S^{2}\right)^{k}$ one obtains in general an exact sequence

$$
0 \rightarrow \mathbb{S}_{n+1}(M) \stackrel{S^{2 k}}{\longrightarrow} \mathbb{S}_{n+2 k+1}(M) \rightarrow H_{n}\left(M, L_{0}(\mathbb{Z})\right) \rightarrow \cdots
$$

where in fact $H_{n}\left(M, L_{0}(\mathbb{Z})\right) \cong H_{n}(M, \mathbb{Z})$; see [26, Remark 25.4].

This near-periodicity can also be defined using products in $L$-theory. Recall the symmetric signature $\sigma^{*}(M)$ of an $n$-dimensional Poincaré complex $M$, a symmetric algebraic Poincaré complex over $\mathbb{Z}$. The products in algebraic surgery [26, Appendix B] give for an $n$-dimensional quadratic algebraic $\mathbb{C}$-Poincaré complex $(C, \psi)$ representing an element in $\mathbb{S}_{n+1}(M)$ a new $(n+4)$-dimensional quadratic algebraic $\mathbb{C}$-Poincaré complex $(C, \psi) \otimes \sigma^{*}\left(\mathbb{C} P^{2}\right)$ representing an element in $\mathbb{S}_{n+5}(M)$. This produces a map which coincides with the double skew-suspension. In geometry this map corresponds to taking a product with the identity on $\mathbb{C} P^{2}$ and projecting algebraically.

More generally one has the following identity of injective homomorphisms

$$
\left(\otimes \sigma^{*}\left(\mathbb{F} P^{2}\right)=S^{k}\right): \mathbb{S}_{n+1}(M) \rightarrow \mathbb{S}_{n+2 k+1}(M)
$$

where $S^{k}$ is $2 k$-skew-suspension map and $\otimes \sigma^{*}\left(\mathbb{F} P^{2}\right)$ is the homomorphism defined by taking the product with the symmetric signature of $\mathbb{C} P^{2}$ or $\mathbb{H} P^{2}$ for $k=2$ or 4 respectively.

\section{Siebenmann periodicity}

Recall that $M$ is a closed topological manifold of dimension $n \geq 5$ and Theorem 1.4 which states that the Cappell-Weinberger map $\mathrm{CW}^{2}: \mathcal{S}(M) \rightarrow \mathcal{S}_{\partial}\left(M \times D^{8}\right)$ is an injective homomorphism with cokernel a subgroup of $\mathbb{Z}$. Theorem 1.4 is a direct consequence of the Proposition 6.1 below. The exactness part follows from the exactness of $S^{4}$ in (5-12) and the identity $\otimes \sigma^{*}\left(\mathbb{H} P^{2}\right)=S^{4}$ of (5-13). 
Proposition 6.1 For $\mathbb{F}=\mathbb{H}$, hence $k=4$, the following diagram commutes.

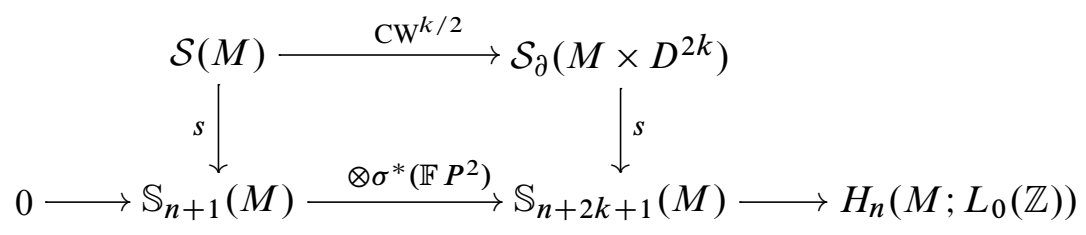

Proof Recall that besides the map $\mathrm{CW}^{k / 2}$ from $\mathcal{S}(M)$ to $\mathcal{S}_{\partial}\left(M \times D^{2 k}\right)$ we have also discussed the extension by a homeomorphism map $E$ which brings us further to $\mathcal{S}\left(M \times \mathbb{F} P^{2}\right)$. This map will be helpful in the proof, in fact the situation can be described by the following diagram:

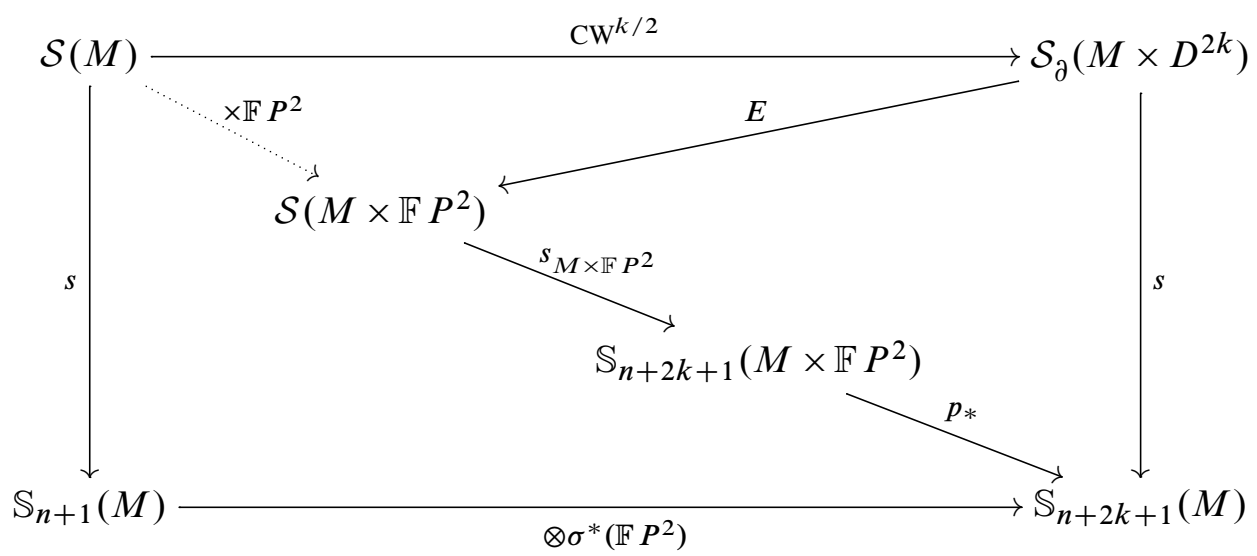

The discussion at the end of the last section shows that the lower triangle commutes. The triangle on the right commutes as well. We warn the reader that we do not claim that the upper triangle commutes, in fact it does not (that's why the arrow is dotted). ${ }^{6}$ Nevertheless we will show that the outer square commutes. For this we first recall Lemma 4.9 which says that $E\left(\mathrm{CW}^{2}([h])\right) \simeq[(h \times \mathrm{id}) \circ \varphi]$ where $\varphi: \bar{N} \rightarrow N \times \mathbb{F} P^{2}$ is a certain homotopy equivalence over the identity of $N$. The proof of the proposition boils down to the following:

Lemma 6.2 For $\mathbb{F}=\mathbb{H}$, hence $k=4$, there is an equality

$$
p_{*}(s([\varphi]))=0 \in \mathbb{S}_{n+2 k+1}(N)
$$

where $p_{*}: \mathbb{S}_{n+2 k+1}\left(N \times \mathbb{F} P^{2}\right) \rightarrow \mathbb{S}_{n+2 k+1}(N)$ is the homomorphism induced by the projection $p: N \times \mathbb{F} P^{2} \rightarrow N$.

\footnotetext{
${ }^{6}$ Just before [11, Lemma 1.4, page 296], Hutt incorrectly stated that the upper triangle commutes. However, it seems from the rest of the paper that this may well have been a typographical error.
} 
We finish the proof of the Proposition 6.1 and then prove Lemma 6.2. We have the following equalities:

$$
\begin{aligned}
s\left(\mathrm{CW}^{k / 2}(h)\right) & =\operatorname{pr}_{1} s\left(E \circ \mathrm{CW}^{k / 2}([h])\right) \\
& =\operatorname{pr}_{1} s([(h \times \mathrm{id}) \circ \varphi]) \\
& =\operatorname{pr}_{1}\left((h \times \mathrm{id})_{*} s([\varphi])+s([h \times \mathrm{id}])\right) \\
& =h_{*} \operatorname{pr}_{1} s([\varphi])+s([h]) \otimes \sigma^{*}\left(\mathbb{F} P^{2}\right) \\
& =s([h]) \otimes \sigma^{*}\left(\mathbb{F} P^{2}\right) .
\end{aligned}
$$

The first equality follows from the definitions and the functoriality of $\mathbb{S}_{\text {? }}(-)$, the second from Lemma 4.9, the third from the composition formula of [27], the fourth again from the functoriality of $\mathbb{S}_{?}(-)$ and the fifth from Lemma 6.2.

Proof of Lemma 6.2 Recall the steps leading from a homotopy equivalence $h: N \rightarrow M$ to the homotopy equivalence $\varphi: \bar{N} \rightarrow N \times \mathbb{F} P^{2}$ defined by (4-11):

(1) Start with a map $\chi: N \rightarrow$ G/TOP (which was chosen so that $(h \times \mathrm{id})^{*} \chi=$ $-[h \times \mathrm{id}] \in[M, \mathrm{G} / \mathrm{TOP}])$.

(2) Construct the homotopy equivalence of pairs $\omega:(\bar{W}, \partial) \rightarrow\left(N \times D^{k+1}, \partial\right)$ over the identity from the $\mathrm{MCN} p:(\bar{W}, \partial) \rightarrow N$ (in Proposition 3.4).

(3) Consider the restriction, $\partial \omega: \partial \bar{W} \rightarrow N \times S^{k}$ which is a homotopy equivalence over the identity.

(4) Construct the homotopy equivalence $\partial \omega^{\prime}: \partial \bar{W}^{\prime} \rightarrow N \times S^{2 k-1}$ over the identity as the pullback of $\gamma_{N}: N \times S^{2 k-1} \rightarrow N \times S^{k}$ along $\partial \omega$ (recall (4-2)). This yields projection maps $q \bar{W}: \partial \bar{W}^{\prime} \rightarrow \partial \bar{W}$ and $p^{\prime}: \partial \bar{W}^{\prime} \rightarrow N$.

(5) Define $\bar{N}=\operatorname{cyl}\left(p^{\prime}\right) \cup \operatorname{cyl}(q \bar{W})$ (Equation (4-10)).

(6) Define $\varphi=\operatorname{cyl}\left(\partial \omega^{\prime}\right.$, id $) \cup \operatorname{cyl}\left(\partial \omega^{\prime}, \partial \omega\right)$ (Equation (4-11)).

Next recall $r: M \rightarrow K$ a homotopy equivalence from $M$ to a $\Delta-$ set $K$ which is transverse to the dual cells of $K$ so that we have a dissection

$$
M=\bigcup_{\sigma \in K} M(\sigma)
$$

with $M(\sigma)=r^{-1}(D(\sigma, K))$ a submanifold with boundary of dimension $(n-|\sigma|)$. Further assume that $h: N \rightarrow M$ is transverse to $M(\sigma)$ for each $\sigma$ so that $N(\sigma)=$ $h^{-1}(M(\sigma))$ is a submanifold with boundary of dimension $(n-|\sigma|)$ and $h(\sigma): N(\sigma) \rightarrow$ $M(\sigma)$ is a degree one normal map. We obtain a dissection

$$
N=\bigcup_{\sigma \in K} N(\sigma) \text {. }
$$


Geometry We show that by a small homotopy it is possible to change $\chi: N \rightarrow \mathrm{G} / \mathrm{TOP}$ so that $\bar{N}$ possess a dissection indexed by simplices $\sigma \in K$, the map $p$ respects the dissections of $\bar{N}$ and $N$ :

$$
\bar{p}=\bigcup \bar{p}(\sigma): \bar{N}=\bigcup \bar{N}(\sigma) \rightarrow N=\bigcup N(\sigma)
$$

and the homotopy equivalence $\varphi: \bar{N} \rightarrow N \times \mathbb{F} P^{2}$ also respects these dissections. Furthermore for each $\sigma \in K$,

$$
\varphi(\sigma): \bar{N}(\sigma) \rightarrow N(\sigma) \times \mathbb{F} P^{2}
$$

is a homotopy equivalence.

To this end modify the map $\chi$ by a small homotopy so that when restricted to the collar of each manifold with boundary $(N(\sigma), \partial N(\sigma))$ it is the product map with the identity in the collar direction. Hence we have

$$
\chi=\bigcup \chi(\sigma): N=\bigcup N(\sigma) \rightarrow \mathrm{G} / \mathrm{TOP} .
$$

To achieve this we follow steps (1)-(6) above but in the relative setting. Note that this requires Proposition 3.5 instead of Proposition 3.4 in step (2). We will proceed inductively starting with simplices $\sigma$ of the top dimension, since then $N(\sigma)$ has dimension 0 . Over such $N(\sigma)$, steps (1) to (6) are trivial. To make the inductive step note that as $k=4$ the dimension restrictions Proposition 3.5 are fulfilled, since by the inductive assumption the dimension of $N(\sigma)$ is $\geq 1$. Steps (3) to (6) have straightforward generalizations to the relative case.

The manifold $\bar{N}$ is the union of all the manifolds $\hat{N}(\sigma)$ just constructed and the projection map $\bar{p}: \bar{N} \rightarrow N$ is the union of the corresponding projections maps $\bar{p}(\sigma)$. Similarly the homotopy equivalence $\varphi$ is the union of all the homotopy equivalences $\varphi(\sigma)$.

Algebraic surgery The homotopy equivalence $\varphi$ represents an element in the structure set $\mathcal{S}\left(N \times \mathbb{F} P^{2}\right)$. The map $s: \mathcal{S}\left(N \times \mathbb{F} P^{2}\right) \rightarrow \mathbb{S}_{n+2 k+1}\left(N \times \mathbb{F} P^{2}\right)$ was described in Section 5. To use it we need to choose a $\Delta$-set homotopy equivalent to $N \times \mathbb{F} P^{2}$. Since $\mathbb{F} P^{2}$ is a triangulable manifold we can choose a triangulation and denote the underlying $\Delta$-set by $L$, the reference map will be denoted $r^{\prime}: \mathbb{F} P^{2} \rightarrow L$. Then we can pick as our choice the geometric product $K \otimes L$, whose geometric realization we identify with the product $|K| \times|L|$, and the reference map $\bar{r}:=(h \circ r) \times r^{\prime}: N \times \mathbb{F} P^{2} \rightarrow|K| \times|L|$ is automatically transverse to the dual cells of $K \otimes L$ which we consider as the underlying space of the geometric product of $\Delta$-sets described in Section 5.

Note that each dual cell of $K \otimes L$ is a subspace of $D(\sigma) \times L$ for suitable $\sigma \in K$. Hence also

$$
\left(N \times \mathbb{F} P^{2}\right)(\sigma, \tau, \lambda) \subset N(\sigma) \times \mathbb{F} P^{2}
$$


for each $\tau, \lambda$. In fact

$$
\bigcup_{\tau, \lambda}\left(N \times \mathbb{F} P^{2}\right)(\sigma, \tau, \lambda) \subset N(\sigma) \times \mathbb{F} P^{2} .
$$

To determine a representative of $s([\varphi])$ in $\mathbb{S}_{n+2 k+1}\left(N \times \mathbb{F} P^{2}\right)$, where we work over the category $\mathbb{Z}_{*}(K \otimes L)$, the map $\varphi$ needs to be made transverse to the submanifolds $\left(N \times \mathbb{F} P^{2}\right)(\sigma, \tau, \lambda)$ for each $(\sigma, \tau, \lambda) \in K \otimes L$. This can be done by a small homotopy which does not spoil the property that $\varphi$ respects the dissections of $\bar{N}$ and $N \times \mathbb{F} P^{2}$ over $K$ and that each $\varphi(\sigma)$ is a homotopy equivalence. To achieve this we can again proceed inductively starting from the simplices $\sigma \in K$ of the top dimension. We change each $\varphi(\sigma)$ by a small homotopy to make it transverse to $\left(N \times \mathbb{F} P^{2}\right)(\sigma, \tau, \lambda)$ for all choices of $\tau$ and $\lambda$, which if course does not spoil the fact that it is a homotopy equivalence. Hence the new $\varphi$ is the union of the new $\varphi(\sigma)$ and hence is transverse to $\left(N \times \mathbb{F} P^{2}\right)(\sigma, \tau, \lambda)$ for each $(\sigma, \tau, \lambda) \in K \otimes L$.

Now we find ourselves in the situation described in Example 5.12. We have the homotopy equivalence $\varphi: \bar{N} \rightarrow N \times \mathbb{F} P^{2}$ whose image $s([\varphi]) \in \mathbb{S}_{n+2 k+1}\left(N \times \mathbb{F} P^{2}\right)$ is represented by a quadratic chain complex over the category $\mathbb{Z}_{*}(K \otimes L)$ whose value at each $(\sigma, \tau, \lambda) \in K \otimes L$ has its underlying chain complex the mapping cone of the Umkehr map of the degree one normal map

$$
\varphi(\sigma, \tau, \lambda): \bar{N}(\sigma, \tau, \lambda)=\varphi^{-1}\left(N \times \mathbb{F} P^{2}\right)(\sigma, \tau, \lambda) \rightarrow\left(N \times \mathbb{F} P^{2}\right)(\sigma, \tau, \lambda) .
$$

This may very well be a representative of a nonzero element in $\mathbb{S}\left(N \times \mathbb{F} P^{2}\right)$.

But we are really interested in the projection $p_{*}(s([\varphi])) \in \mathbb{S}_{n+2 k+1}(N)$. By Example 5.12 this is represented by a quadratic chain complex over the category $\mathbb{Z}_{*}(K)$ whose value at each $\sigma \in K$ has its underlying chain complex the mapping cone of the Umkehr map of the degree one normal map

$$
\varphi(\sigma): \bar{N}(\sigma) \rightarrow N(\sigma) \times \mathbb{F} P^{2} .
$$

But $\varphi(\sigma)$ is a homotopy equivalence for each $\sigma$ and so the resulting chain complex over each $\sigma$ is contractible. Thus by Proposition $5.13 p_{*}(s([\varphi]))=0$ as required.

Remark 6.3 The above proof shows why we chose $k=4$. If $k=2$, then the dimension restrictions of Proposition 3.5 are not satisfied. ${ }^{7}$

\footnotetext{
${ }^{7}$ It is possible that the dimension restrictions in the relevant proposition can be relaxed. This would require careful analysis of all the tools used in the proofs. This might be an interesting problem but lies beyond the scope of this paper.
} 


\section{The bordism groups $\Omega_{2 d-1}^{\mathrm{STOP}}(G / \mathrm{TOP} \times B G)$}

Let $X$ be a space and let $\Omega_{n}^{\text {STOP }}(X)$ denote the $n$-th oriented topological bordism group of $X$. Recall that $G$ is a finite group and $B G$ is its classifying space. The purpose of this section is to prove the following:

Lemma 7.1 For all $d \geq 1, \Omega_{2 d-1}^{\mathrm{STOP}}(\mathrm{G} / \mathrm{TOP} \times B G) \otimes \mathbb{Q}=0$.

Proof The functor $X \rightarrow \Omega_{*}^{\mathrm{STOP}}(X) \otimes \mathbb{Q}$ is a generalised homology theory with coefficients $\Omega_{*}^{\mathrm{STOP}} \otimes \mathbb{Q}$. By Theorem 2.1 for the trivial group $\Omega_{2 d-1}^{\mathrm{STOP}}(\mathrm{pt}) \otimes \mathbb{Q}=0$. To compute $\Omega_{*}^{\mathrm{STOP}}(X)$ applying the Atiyah-Hirzebruch spectral sequence,

$$
\bigoplus_{p+q=*} H_{p}\left(X ; \Omega_{q}^{\mathrm{STOP}} \otimes \mathbb{Q}\right) \Longrightarrow \Omega_{*}^{\mathrm{STOP}}(X) \otimes \mathbb{Q},
$$

we see that if $H_{2 d-1}(X ; \mathbb{Q}) \cong 0$ for all $d$ then $\Omega_{2 d-1}^{\mathrm{STOP}}(X) \otimes \mathbb{Q}=0$ for all $d$.

Now applying [15, Remark 4.36] the space G/TOP is rationally a product of EilenbergMac Lane spaces $K(\mathbb{Q}, 4 i)$ for $i \geq 1$ and so $H_{2 d-1}(\mathrm{G} / \mathrm{TOP} ; \mathbb{Q})=0$ for all $d$. As $G$ is a finite group $H_{*}(B G ; \mathbb{Q})=0$ for all $*>0$ and so by the Künneth Theorem we see that $H_{2 d-1}(\mathrm{G} / \mathrm{TOP} \times B G ; \mathbb{Q})=0$ for all $d$. Thus we conclude that $\Omega_{2 d-1}^{\mathrm{STOP}}(\mathrm{G} / \mathrm{TOP} \times B G) \otimes \mathbb{Q}=0$ for all $d$.

\section{Completion of the proof of Theorem 1.1}

In this section we prove Theorem 1.5 which completes the proof of Theorem 1.1. Recall the definition of the maps $\tilde{\rho}, \tilde{\rho}_{\partial}$ and the $\mathrm{CW}^{k / 2}$-map and that $k=2$ or 4 . In addition recall the map defined by extension by a homeomorphism $E: \mathcal{S}_{\partial}\left(M \times D^{2 k}\right) \rightarrow$ $\mathcal{S}\left(M \times \mathbb{F} P^{2}\right)$. Let $h: N \rightarrow M$ represent an element in $\mathcal{S}(M)$, let $h^{\prime}: N^{\prime} \rightarrow M \times D^{2 k}$ represent $\mathrm{CW}^{k / 2}([h])$. Recall from Lemma 4.9 that $E\left(\left[h^{\prime}\right]\right)$ can be represented by two homotopy equivalences, namely either by $\hat{h}: \hat{N} \rightarrow M \times \mathbb{F} P^{2}$ (see (4-9)) or by $\bar{h}: \bar{N} \rightarrow M \times \mathbb{F} P^{2}$ (see just below (4-11)).

Lemma 8.1 There are identities

(1) $\rho(\bar{N})=\tilde{\rho}_{\partial}\left(\left[h^{\prime}\right]\right)+\rho(M)$,

(2) $\rho(\bar{N})=\rho(N)$.

Proof of Theorem 1.5 Combining Lemma 8.1 (1) and (2) we have

$$
\tilde{\rho}_{\partial}\left(\left[h^{\prime}\right]\right)=\rho(N)-\rho(M)=\tilde{\rho}([h]) .
$$


Proof of Lemma 8.1 (1) The argument is similar to the proof of Proposition 2.9. From the rel. boundary structure $h^{\prime}: N^{\prime} \rightarrow M \times D^{k}$ we form the closed manifold $M\left(h^{\prime}\right):=N^{\prime} \cup_{h^{\prime}}\left(-M \times D^{2 k}\right)$ and by Definition 2.6, $\tilde{\rho}_{\partial}\left(\left[h^{\prime}\right]\right)=\rho\left(M\left(h^{\prime}\right)\right)$. Recall the operation $\#_{M}$ in Definition 2.7 and observe that

$$
M \times \mathbb{F} P^{2}=\left(M \times \mathbb{F} P^{2 \bullet}\right) \cup\left(M \times D^{2 k}\right) .
$$

If follows that we can form the closed manifold

$$
M\left(h^{\prime}\right) \#_{M}\left(M \times \mathbb{F} P^{2}\right)
$$

just as in Definition 2.7. By the same reasoning as in the proof of Proposition 2.9 we obtain that the $\rho$-invariant of the manifold in (8-1) is the sum of $\rho$-invariants $\rho\left(M\left(h^{\prime}\right)\right)+\rho\left(M \times \mathbb{F} P^{2}\right)$. But from the construction of $\hat{N}$ in (4-8) we see that there is a homeomorphism

$$
\widehat{N} \cong M\left(h^{\prime}\right) \#_{M}\left(M \times \mathbb{F} P^{2}\right) .
$$

The statement now follows since $\rho\left(M \times \mathbb{F} P^{2}\right)=\rho(M)$ and $\rho(\hat{N})=\rho(\bar{N})$, which we have from the $h$-cobordism invariance of the $\rho$-invariant.

(2) We are given $[h: N \rightarrow M] \in \mathcal{S}(M)$ and $\left[\bar{h}: \bar{N} \rightarrow M \times \mathbb{F} P^{2}\right] \in \mathcal{S}\left(M \times \mathbb{F} P^{2}\right)$ which represents $E \circ \mathrm{CW}^{k / 2}([h])$ and we wish to prove that $\rho(\bar{N})=\rho(N)$ where the reference map for $\bar{N}$ is $\lambda(N) \circ \bar{p}$ and $\bar{p}: \bar{N} \rightarrow N$ is the map constructed in Section 4.2.

Recall from Definition 2.2 the definition of the $\rho$-invariant of a $(2 d-1)$-dimensional manifold $N$ equipped with a map $\lambda(N): N \rightarrow B G$ inducing $\lambda(N)_{*}: \pi_{1}(N) \rightarrow G$, with $G$ a finite group. Because $\Omega_{2 d-1}^{\text {STOP }}(B G) \otimes \mathbb{Q}=0$ there is a coboundary for $\bigsqcup_{i=1}^{r} N$ over $\lambda(N)$ for some $r \geq 1$. That is, there is a manifold $P$ with boundary $\partial P=\bigsqcup_{i=1}^{r} N$, and with a map $\lambda(P): P \rightarrow B G$ extending $\bigsqcup^{r} \lambda(N)$. The formula is

$$
\rho(N):=(1 / r) G-\operatorname{sign}(P) .
$$

To show the desired statement it is enough to find a coboundary, say $\bar{P}$, for $\bigsqcup_{i=1}^{r} \bar{N}$ over $\lambda(\bar{N})=(\lambda(N) \circ \bar{p}): \bar{N} \rightarrow N \rightarrow B G$, such that $\bar{P} \simeq P \times \mathbb{F} P^{2}$. Then by the multiplicativity of $G$-signature we would obtain

$$
\rho(\bar{N})=(1 / r) G-\operatorname{sign}(\bar{P})=(1 / r) G-\operatorname{sign}(P) \cdot \operatorname{sign}\left(\mathbb{F} P^{2}\right)=\rho(N) .
$$

Recall from Section 4 that the closed manifold $\bar{N}$ along with a homotopy equivalence $\varphi: \bar{N} \rightarrow N \times \mathbb{F} P^{2}$ was constructed from a map $\chi(N): N \rightarrow \mathrm{G} / \mathrm{TOP}$. Now, by Lemma 7.1 we have $\Omega_{2 d-1}^{\mathrm{STOP}}(B G \times \mathrm{G} / \mathrm{TOP}) \otimes \mathbb{Q}=0$. This implies that there exists a 
manifold $P$ with boundary $\partial P=\bigsqcup_{i=1}^{r} N$ and a map

$$
\kappa(P): P \rightarrow B G \times \mathrm{G} / \mathrm{TOP}
$$

such that

$$
\operatorname{pr}_{\mathrm{G} / \mathrm{TOP}} \circ\left(\left.\kappa(P)\right|_{\partial P}\right)=\bigsqcup_{i=1}^{r} \chi(N):\left(\bigsqcup_{i=1}^{r} N\right) \rightarrow \mathrm{G} / \mathrm{TOP}
$$

and

$$
\operatorname{pr}_{B G} \circ\left(\left.\kappa(P)\right|_{\partial P}\right)=\bigsqcup_{i=1}^{r} \lambda(N):\left(\bigsqcup_{i=1}^{r} N\right) \rightarrow B G .
$$

Here $\mathrm{pr}_{\mathrm{G} / \mathrm{TOP}}$ and $\mathrm{pr}_{B G}$ are the obvious projections. We have used the same letter $P$ as above because such a $P$ can be used as a coboundary of $\bigsqcup_{i=1}^{r} N$ in (8-2). The improvement is that now $P$ comes equipped with the composite map $\chi(P):=$ $\mathrm{pr}_{\mathrm{G} / \mathrm{TOP}} \circ \kappa(P): P \rightarrow \mathrm{G} / \mathrm{TOP}$.

Recall the recipe for constructing $\bar{N}$ from $\chi(N): N \rightarrow \mathrm{G} /$ TOP repeated in the proof of Lemma 6.2 as steps (1) to (6). In that proof a generalization of steps (1) to (6) was used when one starts with a map from a manifold with boundary to G/TOP.

Using this generalised procedure we construct a manifold with boundary $\bar{P}$ with a homotopy equivalence $\varphi(P): \bar{P} \rightarrow P \times \mathbb{F} P^{2}$. The boundary is $\partial \bar{P}=\bigsqcup_{i=1}^{r} \bar{N}$ since the map $\chi(P): P \rightarrow \mathrm{G} / \mathrm{TOP}$, restricts to $\bigsqcup_{i=1}^{r} \chi(N): \bigsqcup_{i=1}^{r} N \rightarrow \mathrm{G} / \mathrm{TOP}$ on $\partial P=\bigsqcup_{i=1}^{r} N$. Furthermore if $\bar{p}(P): \bar{P} \rightarrow P$ denotes the analogue of $\bar{p}: \bar{N} \rightarrow N$ obtain from the generalised procedure, then we have the map

$$
\lambda(\bar{P})=(\lambda(P) \circ \bar{p}(P)): \bar{P} \rightarrow P \rightarrow B G,
$$

which restricts to $\bigsqcup_{i=1}^{r} \lambda(\bar{N})$ on the boundary. If follows that $\bar{P}$ is the desired coboundary of $\bigsqcup_{i=1}^{r} \bar{N}$ over $\lambda(\bar{N})$ which may be used in (8-3).

\section{References}

[1] MF Atiyah, R Bott, A Lefschetz fixed point formula for elliptic complexes. I, Ann. of Math. (2) 86 (1967) 374-407 MR0212836

[2] MF Atiyah, I M Singer, The index of elliptic operators. III, Ann. of Math. (2) 87 (1968) 546-604 MR0236952

[3] G Brumfiel, Homotopy equivalences of almost smooth manifolds, Comment. Math. Helv. 46 (1971) 381-407 MR0305419

[4] S Cappell, S Weinberger, A geometric interpretation of Siebenmann's periodicity phenomenon, from: "Geometry and topology (Athens, Ga., 1985)", (C McCrory, T Shifrin, editors), Lecture Notes in Pure and Appl. Math. 105, Dekker, New York (1987) 47-52 MR873283 
[5] T A Chapman, Approximation results in Hilbert cube manifolds, Trans. Amer. Math. Soc. 262 (1980) 303-334 MR586720

[6] P E Conner, E E Floyd, Differentiable periodic maps, Ergebnisse der Math. und ihrer Grenzgebiete 33, Academic Press, New York (1964) MR0176478

[7] J F Davis, W Lück, Manifolds homotopy equivalent to certain torus bundles over lens spaces, in preparation (2010)

[8] M H Freedman, F Quinn, Topology of 4-manifolds, Princeton Math. Series 39, Princeton Univ. Press (1990) MR1201584

[9] B Hughes, Stratifications of mapping cylinders, Topology Appl. 94 (1999) 127-145 MR1695351 Special issue in memory of B J Ball

[10] B Hughes, L R Taylor, E B Williams, Bundle theories for topological manifolds, Trans. Amer. Math. Soc. 319 (1990) 1-65 MR1010410

[11] S Hutt, On Siebenmann periodicity, Pacific J. Math. 183 (1998) 291-303 MR1625974

[12] B Jahren, S Kwasik, Free involutions on $S^{1} \times S^{n}$, to appear in Math. Ann. arXiv: 0802.2035

[13] R C Kirby, L C Siebenmann, Foundational essays on topological manifolds, smoothings, and triangulations, Annals of Math. Studies 88, Princeton Univ. Press (1977) MR0645390 With notes by J Milnor and M Atiyah

[14] T Macko, C Wegner, On the classification of fake lens spaces, to appear in Forum Math. arXiv:0810.1196

[15] I Madsen, R J Milgram, The classifying spaces for surgery and cobordism of manifolds, Annals of Math. Studies 92, Princeton Univ. Press (1979) MR548575

[16] I Madsen, M Rothenberg, On the classification of G-spheres. II. PL automorphism groups, Math. Scand. 64 (1989) 161-218 MR1037458

[17] I Madsen, L R Taylor, B Williams, Tangential homotopy equivalences, Comment. Math. Helv. 55 (1980) 445-484 MR593058

[18] A J Nicas, Induction theorems for groups of homotopy manifold structures, Mem. Amer. Math. Soc. 39, no. 267, Amer. Math. Soc. (1982) MR668807

[19] E K Pedersen, Embeddings of topological manifolds, Illinois J. Math. 19 (1975) 440447 MR0377898

[20] T Petrie, The Atiyah-Singer invariant, the Wall groups $L_{n}(\pi, 1)$, and the function $\left(t e^{x}+1\right) /\left(t e^{x}-1\right)$, Ann. of Math. (2) 92 (1970) 174-187 MR0319216

[21] F Quinn, A geometric formulation of surgery, from: "Topology of Manifolds (Proc. Inst., Univ. of Georgia, Athens, Ga., 1969)", (J C Cantrell, C H Edwards, Jr, editors), Markham, Chicago (1970) 500-511 MR0282375

[22] F Quinn, Ends of maps. I, Ann. of Math. (2) 110 (1979) 275-331 MR549490 
[23] A Ranicki, The total surgery obstruction, from: "Algebraic topology, Aarhus 1978 (Proc. Sympos., Univ. Aarhus, Aarhus, 1978)", (J L Dupont, IH Madsen, editors), Lecture Notes in Math. 763, Springer, Berlin (1979) 275-316 MR561227

[24] A Ranicki, The algebraic theory of surgery. I. Foundations, Proc. London Math. Soc. (3) 40 (1980) 87-192 MR560997

[25] A Ranicki, The algebraic theory of surgery. II. Applications to topology, Proc. London Math. Soc. (3) 40 (1980) 193-283 MR566491

[26] A Ranicki, Algebraic L-theory and topological manifolds, Cambridge Tracts in Math. 102, Cambridge Univ. Press (1992) MR1211640

[27] A Ranicki, A composition formula for manifold structures, Pure Appl. Math. Q. 5 (2009) 701-727 MR2508900

[28] A Ranicki, M Weiss, On the algebraic L-theory of $\Delta$-sets arXiv: math/0701833v2

[29] C P Rourke, B J Sanderson, On topological neighbourhoods, Compositio Math. 22 (1970) 387-424 MR0298671

[30] C P Rourke, B J Sanderson, $\Delta$-sets. I. Homotopy theory, Quart. J. Math. Oxford Ser. (2) 22 (1971) 321-338 MR0300281

[31] L C Siebenmann, Periodicity in topological surgery, from: "Foundational essays on topological manifolds, smoothings, and triangulations", Annals of Math. Studies 88, Princeton Univ. Press (1977) vii+355

[32] C T C Wall, Surgery on compact manifolds, second edition, Math. Surveys and Monogr. 69, Amer. Math. Soc. (1999) MR1687388 Edited and with a foreword by A Ranicki

[33] R E Williamson, Jr, Cobordism of combinatorial manifolds, Ann. of Math. (2) 83 (1966) 1-33 MR0184242

DC: Hausdorff Research Institute for Mathematics

Poppelsdorfer Allee 82, D-53115 Bonn, Germany

TM: Mathematisches Institut, Universität Bonn

Endenicher Allee 60, D-53115 Bonn, Germany

Matematický Ústav SAV

Štefánikova 49, Bratislava SK-81473 Slovakia

diarmuidc23@gmail.com, macko@uni-muenster.de, macko@math.uni-bonn.de http://www.dcrowley.net/, http://www.math.uni-bonn.de/people/macko/

Received: 9 February $2010 \quad$ Revised: 30 March 2011 